ISSN: 0210-1696

DOI: http://dx.doi.org/10.14201/scero2016472741

\title{
EVALUACIÓN DE ACTITUDES \\ DE LOS PROFESIONALES HACIA \\ LAS PERSONAS CON DISCAPACIDAD
}

\section{Assessment of Staff Attitudes towards People with Disability}

\author{
Víctor Arias González \\ Facultad de Psicología. Universidad de Talca. Chile \\ viarias@utalca.cl \\ Benito Arias MartíneZ \\ Facultad de Educación. Universidad de Valladolid \\ Miguel Ángel Verdugo Alonso \\ Facultad de Psicología. Universidad de Salamanca \\ Mariano Rubia Avi \\ Facultad de Educación. Universidad de Valladolid \\ Cristina Jenaro Río \\ Facultad de Psicología. Universidad de Salamanca
}

Recepción: 5 de abril de 2016

Fecha de aceptación definitiva: 25 de mayo de 2016

RESUMEN: El propósito principal de este estudio ha sido la construcción y validación de una escala de actitudes hacia las personas con discapacidad, dirigida preferentemente a profesionales de las áreas de la salud y la educación. Han participado 976 profesionales de diferentes ámbitos (sanidad, educación, orientación laboral, medios de comunicación, administración), con un rango de edad entre 18 y 65 años. El 49.1\% fueron mujeres. Se han realizado análisis de concordancia entre jueces expertos para valorar el contenido de los ítems, y se han sometido los datos a tres clases de análisis: comparación de estructuras de correlación, análisis factorial exploratorio con método de factor común y análisis factorial confirmatorio. Se han comparado dos modelos: unifactorial y de tres factores correlacionados. Los resultados han evidenciado buenas cualidades métricas de fiabilidad 
(consistencia interna, fiabilidad de los indicadores individuales, fiabilidad de constructo y error típico de medida) y de validez (de contenido, convergente y discriminante).

Palabras Clave: actitudes; evaluación; personas con discapacidad; profesionales.

ABSTRACT: The main purpose of this study was the construction and validation of a scale of attitudes towards people with disabilities, mainly directed at professionals in the areas of health and education. Participants were 976 professionals from different fields (health, education, career counseling, media, administration), with an age range between 18 and 65 years. $49.1 \%$ were women. After analyzing agreement between expert judges to evaluate the content of the items, three types of analysis were performed: comparison of correlation structures, exploratory factor analysis by common factor method, and confirmatory factor analysis on polichoric correlations matrix. Two models were compared: Unidimensional and three-correlated factors. Results have shown good metric qualities of reliability (internal consistency, reliability of individual indicators, construct reliability and standard error of measurement) and validity (content, convergent and discriminant).

KEY WORDS: attitudes; assessment; persons with disabilities; staff.

\section{Evaluación de actitudes de los profesionales hacia las personas con discapacidad}

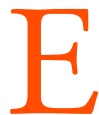

L ÉXITO O FRACASO DE LAS POLÍTICAS tendentes a mejorar la inclusión social de las personas con discapacidad depende en gran medida del grado de conocimiento, las creencias y las actitudes de la sociedad hacia la discapacidad. Cuando las actitudes de la comunidad son negativas y prevalecen las creencias estigmatizantes, los esfuerzos para conseguir la integración, la inclusión, la independencia y la autodeterminación de las personas con discapacidad se encontrarán con serias resistencias (Ali, Strydom, Hassiotis, Williams y King, 2008; Antonak y Livneh, 2000; Scior, 2011; Scior y Furnham, 2011; WHO, 2011). En consecuencia, entender las actitudes hacia la discapacidad tiene importantes implicaciones para la probabilidad de éxito de las políticas inclusivas. Diferentes investigaciones (Akrami, Ekehammer, Claesson y Sonnander, 2006; Arias y Morentin, 2004; Hampton y Xiao, 2007; Lombardi y Murray, 2011; Hatton, Akram, Robertson, Shah y Emerson, 2003; Scior y Werner, 2015; Tachibana y Watanabe, 2003, 2004) ponen de manifiesto que el grado de conocimiento sobre la discapacidad es muy limitado en la sociedad y más escaso de lo que sería deseable en los profesionales que prestan servicios a personas con discapacidad, y que esa falta de información está estrechamente vinculada con actitudes negativas y con creencias estigmatizantes.

A partir de las definiciones -clásicas y más actuales- que se han dado del concepto (e.g., Breckler y Wiggins, 1992; Eagly y Chaiken, 1995; Forgas, Cooper y Crano, 2010; Bohner y Wänke, 2014; Triandis, 1971), podemos sintetizar que las actitudes son construcciones teóricas, que constan de componentes cognitivo, afectivo y conativo-conductual, y que se infieren a partir de la observación de la conducta o de las 
manifestaciones verbales de la persona, puesto que no son por sí mismas directamente constatables. A menudo se definen como constructos psicosociales inferidos o latentes, o como procesos que, se postula, residen dentro del individuo. Además, estos constructos o procesos están bajo el dominio de estímulos específicos u objetos de referencia (por ejemplo, individuos, grupos sociales). Estos referentes, en virtud de su valor social percibido, son capaces de elicitar una respuesta actitudinal por parte del sujeto. En este sentido, las actitudes pueden ser concebidas como mediadores entre un estímulo del entorno y unas respuestas conductuales.

En su intento por desarrollar métodos cada vez más refinados para evaluar las actitudes, los investigadores han ideado multitud de estrategias y procedimientos que, si bien comparten un objetivo similar, difieren en otros aspectos tales como los presupuestos teóricos en los que se apoyan, los formatos utilizados, los niveles de sistematización requeridos, el número de sujetos a los que se aplican simultáneamente, el nivel de precisión exigido en la recogida de información o la naturaleza de los datos recogidos, entre otros (Antonak y Livneh, 1994, 2000). La clasificación más común establece una diferencia entre métodos directos e indirectos.

La característica que define los métodos indirectos es la inferencia o interpretación que el evaluador debe realizar sobre la naturaleza de las actitudes inherentes a las respuestas de los sujetos quienes, en mayor o menor grado según los distintos métodos especificados en la Tabla 1, desconocen tanto el hecho de estar siendo observados o evaluados como, en su caso, el fin de la evaluación. Estos métodos pretenden eludir o minimizar los sesgos (e. g., reactividad, deseabilidad social, falsificación) asociados al conocimiento de estar siendo observado o evaluado. Así, puede desconocer el objeto de la evaluación (observaciones conductuales), ser engañado a propósito respecto al fin de la evaluación (procedimientos solapados o encubiertos) o conocer que está siendo evaluado, pero ignorar el objeto de la evaluación (técnicas proyectivas).

Los métodos directos de evaluación son los más ampliamente conocidos y utilizados, y su característica distintiva frente a los métodos indirectos radica en que los sujetos evaluados conocen que sus actitudes están siendo sometidas a evaluación. Las modalidades difieren tanto en el formato utilizado como en el propósito perseguido. Tampoco estos métodos están exentos de inconvenientes (e. g., sensibilización del respondiente, o sesgos tales como la aquiescencia, la deseabilidad social, el falseamiento consciente de las respuestas o la tendencia a seleccionar puntuaciones medias o extremas). Entre los métodos directos, los más utilizados en la evaluación de actitudes hacia la discapacidad han sido las escalas de clasificación ("rating scales"), probabilísticas de estimaciones sumatorias o "tipo Likert". Mediante estas escalas se pretende obtener una evaluación o juicio cuantitativo basados en juicios personales. La respuesta de la persona evaluada indica la intensidad de su acuerdo o desacuerdo con cada ítem relativo al referente de la actitud. El formato de respuesta puede ser gráfico, numérico o verbal. Independientemente del formato que se adopte para las respuestas, éstas están completamente estructuradas de antemano por el investigador. Por tanto, las personas evaluadas no tienen oportunidad de añadir otros juicios o comentarios, o de responder a los ítems de forma diferente a la especificada en la escala de respuesta. 
EVALUACIÓN DE ACTITUDES DE LOS PROFESIONALES HACIA LAS PERSONAS CON DISCAPACIDAD

V. ARIAS GONZÁLEZ, B. ARIAS MARTÍNEZ, M. Á. VERDUGO ALONSO, M. RUBIA AVI Y C. JENARO RÍO

\begin{tabular}{|c|c|c|c|c|c|}
\hline Referencia & Instrumento & Tipo & Referente & Dim. & Dest. \\
\hline Akrami et al. (2006) & MCA & ES & $G$ & 2 & $\mathrm{~A}$ \\
\hline Antonak $(1979,1981)$ & SADP & ES & $G$ & 3 & $\mathrm{~A}$ \\
\hline Antonak (1985) & ATDS & IAI & $\mathrm{E}$ (Varios)* & ¿? & $\mathrm{A}$ \\
\hline Antonak \& Rankin (1982) & ATPE & ES & E (Epileps.) & 3 & $\mathrm{~A}$ \\
\hline Arias et al. (1995) & $\mathrm{APD}$ & $\mathrm{ES}$ & $\mathrm{G}, \mathrm{E}$ & 3 & $\mathrm{~A}$ \\
\hline Bagley \& Greene (1981) & PATHS & IAI & $\mathrm{E}$ (Integr.) & ¿? & $\mathrm{N}$ \\
\hline Bartlett et al. (1960) & AMRP & ES & $\mathrm{E}(\mathrm{DI})$ & ¿? & $\mathrm{A}$ \\
\hline Berryman \& Neal (1980) & ATMS & $\mathrm{ES}$ & $\mathrm{E}$ (Integr.) & 3 & $\mathrm{~A}$ \\
\hline Campbell (2008) & SAQ & ES & G & 3 & $\mathrm{~A}$ \\
\hline Chadd \& Pangilian (2011) & DAHC & $\mathrm{ES}$ & $G$ & ¿? & $\mathrm{A}$ \\
\hline Clayfield et al. (2011) & MHASP & ES & $\mathrm{E}(\mathrm{EM})$ & 4 & $\mathrm{~A}$ \\
\hline Cohen \& Struening $(1959,1960)$ & OMI & $\mathrm{ES}$ & E (P. Psiq.) & 5 & $\mathrm{~A}$ \\
\hline Cohen \& Struening (1962) & OMI & ES & $\mathrm{E}(\mathrm{EM})$ & 5 & $\mathrm{~A}$ \\
\hline Cooper et al. (2004) & ADS & ES & E (Sordera) & 6 & $\mathrm{~A}$ \\
\hline Cowen et al. (1958) & $\mathrm{AB}$ & $\mathrm{ES}$ & E (Ceguera) & ¿? & $\mathrm{A}$ \\
\hline Cowen et al. (1967) & $\mathrm{AD}$ & ES & E (Sordera) & ¿? & $\mathrm{A}$ \\
\hline Cowen et al. (1967) & ADS & ES & E (Sordera) & 5 & $\mathrm{~A}$ \\
\hline Efron \& Efron (1967) & ATR & ES & $\mathrm{E}(\mathrm{DI})$ & 6 & $\mathrm{~A}$ \\
\hline Esposito \& Peach (1983) & PSSHP & ENT & G & ¿? & $\mathrm{N}$ \\
\hline Fellinghauer et al. (2011) & PESD & ES & $\mathrm{G}$ & ¿? & $\mathrm{A}$ \\
\hline Fiedler \& Simpson (1987) & STJCBAS & ES & G & ¿? & $\mathrm{A}$ \\
\hline Findler et al. (2007) & MDIAT & $\mathrm{ES}$ & $G$ & ¿? & $\mathrm{A}$ \\
\hline Gan et al. (1977) & MRQ & ES & $\mathrm{E}(\mathrm{DI})$ & 8 & $\mathrm{~A}$ \\
\hline Gilbert \& Levinson (1956) & $\mathrm{CMI}$ & $\mathrm{ES}$ & E (P. Psiq.) & ¿? & $\mathrm{A}$ \\
\hline Grand et al. (1982) & DSRS & $\mathrm{ES}$ & $\mathrm{E}($ Varios) $* *$ & ¿? & $\mathrm{A}$ \\
\hline Groomes \& Linkowski (2007) & ADS-R & ES & $\mathrm{G}$ & 4 & $\mathrm{~A}(\mathrm{DI})$ \\
\hline Hampton \& Xiao (2008) & MRAI-R & $\mathrm{ES}$ & $\mathrm{E}(\mathrm{DI})$ & 4 & $\mathrm{~A}$ \\
\hline Harth (1974) & MRAI & ES & $\mathrm{E}(\mathrm{DI})$ & 3 & $\mathrm{~A}$ \\
\hline Henry et al. (1996) & CLAS-MR & $\mathrm{ES}$ & E (Incl.Soc.) & 4 & $\mathrm{~A}$ \\
\hline Horne (1985) & PCS & ES & $G$ & ¿? & $\mathrm{A}$ \\
\hline Kassam et al. (2010) & MICA & ES & $\mathrm{E}(\mathrm{EM})$ & $¿ ?$ & $\mathrm{~A}$ \\
\hline Kiertsch et al. (1980) & CADS & $\mathrm{ES}$ & G (Integr.) & ¿? & $\mathrm{N}$ \\
\hline Langevin \& Hagler (2004) & PATCS & ES & $\mathrm{E}$ (Comun.) & 3 & $\mathrm{~N}$ \\
\hline Larrivee \& Cook (1979) & ARM & ES & G (Integr.) & 5 & $\mathrm{~A}$ \\
\hline Lazar et al. (1971) & ATHI & ES & $\mathrm{G}$ & 1 & $\mathrm{~A}$ \\
\hline Lidsley \& Frith (1983) & CATDP & ES & $\mathrm{G}$ (Integr.) & 1 & $\mathrm{~N}$ \\
\hline Lilienfeld \& Alant (2002) & CADAQ & $\mathrm{ES}$ & E (Sordera) & 3 & $\mathrm{~N}$ \\
\hline Livneh (1985) & DFS-G & ES & $G$ & 7 & $\mathrm{~A}$ \\
\hline
\end{tabular}

() Ediciones Universidad de Salamanca / CC BY-NC-ND

Siglo Cero, vol. 47 (2), n. ${ }^{\circ} 258,2016$, abril-junio, pp. 7-41 


\begin{tabular}{|c|c|c|c|c|c|}
\hline Referencia & Instrumento & Tipo & Referente & Dim. & Dest. \\
\hline Marsh \& Friedman (1972) & ABS & ES & E (Ceguera) & ¿? & $\mathrm{A}$ \\
\hline Miller et al. (1981) & SCATE & $\mathrm{PRO}$ & $\mathrm{G}$ & 3 & $\mathrm{~N}$ \\
\hline Morin et al. (2013) & ATTID & ES & $\mathrm{E}(\mathrm{DI})$ & 3 & $\mathrm{~A}$ \\
\hline Nunnally (1961) & MIHIQ & $\mathrm{ES}$ & E (P. Psiq.) & 10 & $\mathrm{~A}$ \\
\hline Parish et al. (1978) & PAIC & LA & $\mathrm{G}$ (Integr.) & ¿? & $\mathrm{N}$ \\
\hline Popovich et al. (2003) & DQ & ES & G & ¿? & $\mathrm{A}$ \\
\hline Power et al. (2010) & ADS-P & ES & $\mathrm{E}(\mathrm{DI})$ & 4 & $\mathrm{~A}(\mathrm{DI})$ \\
\hline Power et al. (2010) & ADS-G & ES & $\mathrm{E}(\mathrm{DI})$ & 4 & $\mathrm{~A}(\mathrm{G})$ \\
\hline Pruett \& Chan (2006) & DA-IAT & $\mathrm{ES}$ & $G$ & ¿? & $\mathrm{A}$ \\
\hline Reynolds \& Greco (1980) & EAS & $\mathrm{ES}$ & G (Integr.) & 2 & $\mathrm{~A}$ \\
\hline Rosenbaum et al. (1985) & CATCH & ES & $\mathrm{G}$ & 3 & $\mathrm{~A}$ \\
\hline Rucker \& Gable (1974) & RGEPS & IAI & G (Integr.) & 4 & $\mathrm{~A}$ \\
\hline Saunders et al. (2005) & ALHQ & ES & E (Sordera) & 4 & $\mathrm{~A}$ \\
\hline \begin{tabular}{|l|} 
Schmelkin (1981) \\
\end{tabular} & $\mathrm{MO}$ & $\mathrm{ES}$ & G (Integr.) & 2 & $\mathrm{~A}$ \\
\hline Scior (2011) & IDLS & ES & $G$ & 4 & $\mathrm{~A}$ \\
\hline Siller et al. (1967) & DFS & ES & $\mathrm{G}$ & 7 & $\mathrm{~A}$ \\
\hline Symons et al. (2012) & MSA & ES & $\mathrm{G}$ & 3 & $\mathrm{~A}$ \\
\hline Tachibana \& Watanabe (2003) & $\mathrm{DQ}$ & $\mathrm{ES}$ & $\mathrm{G}$ & ¿? & $\mathrm{A}$ \\
\hline Taylor \& Dear (1981) & CAMI & $\mathrm{ES}$ & $\mathrm{E}(\mathrm{EM})$ & 4 & $\mathrm{~A}$ \\
\hline Thomas et al. (2002) & IDP & ES & $\mathrm{G}$ & ¿? & $\mathrm{A}$ \\
\hline Towfighy-Hoshyar \& Zingle (1984) & PAS & ES & $\mathrm{E}$ (Integr.) & 4 & $\mathrm{~N}$ \\
\hline Tringo (1970) & DSDS & IAI & $E$ & 1 & $\mathrm{~A}$ \\
\hline Upton \& Harper (2002) & MDA & $\mathrm{ES}$ & E (E.S.) & 2 & $\mathrm{~A}$ \\
\hline Verdugo, Arias y Jenaro (1994) & $\mathrm{APD}$ & $\mathrm{ES}$ & $\mathrm{G} / \mathrm{E}^{* * *}$ & 5 & $\mathrm{~A}$ \\
\hline \begin{tabular}{|l} 
Voeltz (1980) \\
\end{tabular} & A-S & ES & G & 4 & $\mathrm{~N}$ \\
\hline Willems et al. (2010) & SCIBI & ES & $\mathrm{E}(\mathrm{DI})$ & 4 & $\mathrm{~A}$ \\
\hline \begin{tabular}{|l|} 
Williams \& Rose (2007) \\
\end{tabular} & $\mathrm{CBPQ}$ & ES & E (P.Cond.) & 6 & $\mathrm{~A}$ \\
\hline Yuker et al. (1960) & ATDP & ES & $\mathrm{G}$ & 1 & $\mathrm{~A}$ \\
\hline
\end{tabular}

Notas. Instrumentos: ES = Estimaciones sumatorias; IAI = Intervalos Aparentemente Iguales; PRO = Proyectivas; $\mathrm{LA}=$ Lista de Adjetivos; ENT = Entrevista. Referente: $\mathrm{E}=$ Específica; $\mathrm{G}=$ General.

Destinatario: $\mathrm{A}=$ Adulto; $\mathrm{N}=$ Niño.

* Parálisis cerebral, epilepsia, amputación, ceguera.

* Enfermedad crónica, problemas emocionales, enfermedad mental, discapacidad física, discapacidad sensorial.

** Discapacidad física, discapacidad sensorial, discapacidad intelectual.

En lo que concierne a los instrumentos de evaluación de las actitudes hacia las personas con discapacidad, cabe decir que cuentan con una larga historia que se inicia en los años 60 del siglo pasado y continúa hasta nuestros días, si bien en no pocas ocasiones se trata de estudios basados en muestras pequeñas o no representativas de la población de referencia, o de estudios transversales meramente descriptivos 
y no replicados (Scior, 2011). En la Tabla 1 se muestran algunos de los principales instrumentos desarrollados. Como se ve, se incluyen métodos directos y algunos indirectos, pero predominan claramente las escalas de estimaciones sumatorias. En la revisión realizada por Antonak y Livneh (2000) se concluye que la evaluación de actitudes mediante dichas escalas puede estar afectada por el sesgo de la deseabilidad social, por lo que se requiere el desarrollo de métodos innovadores de evaluación e instrumentos que demuestren suficiente solidez psicométrica en cuando a las evidencias de validez, fiabilidad, dimensionalidad y sensibilidad al cambio. Si carecemos de tales instrumentos, no será posible obtener respuestas concluyentes a importantes preguntas de investigación que tienen que ver con la relación entre las actitudes y la aceptación, la integración y la inclusión plena de las personas con discapacidad en la sociedad (Bailey, Barr y Bunting, 2001; Bigby, Clement, Mansell y Beadle-Brown, 2009; Edwards, Lennox y White, 2007; Graham, Herbert, Price y Williams, 2004; Ostapczuk y Musch, 2011). En el caso de la evaluación de actitudes hacia las personas con discapacidad en lengua española, carecemos de instrumentos actualizados y validados con muestras españolas representativas (y menos aún con instrumentos específicamente dirigidos a profesionales) y desarrollados con estándares elevados de calidad científica, por lo que el objetivo del presente estudio se ha centrado en la validación y aplicación de un instrumento con propiedades métricas sólidas, centrado en la evaluación de las actitudes de los profesionales (esencialmente encuadrados en los ámbitos de la sanidad y la educación) hacia las personas con discapacidad.

\section{Método}

\section{Participantes}

En el estudio participaron 976 profesionales pertenecientes a los ámbitos de la sanidad, educación, orientación laboral, medios de comunicación, policía municipal, administración autonómica, administración local, CEAS, oficinas de empleo, oficinas de información, coordinadores de diferentes Direcciones Generales de la Junta de Castilla y León (Función Pública, Administración Territorial, Comunicación, Estadística, etc.), justicia y transporte de las 9 provincias de la Comunidad Autónoma de Castilla y León. El 49.1\% fueron mujeres. El rango de edad estuvo comprendido entre 18 y 65 años. El 71.7\% de los respondientes tenían entre 31 y 50 años, predominando dentro de este grupo aquellos profesionales con edades comprendidas entre los 41 y los 50 años (39.6\%). El 6.4\% declaró haber alcanzado estudios primarios, el $5.4 \%$ bachillerato, el $26.4 \%$ universitarios medios y el $61.8 \%$ universitarios superiores. El 78.9\% de los participantes manifestó tener contacto directo con al menos una persona con discapacidad. El tipo de contacto más frecuente fue el laboral (55.9\%). Se observó menor frecuencia en otras clases de contacto: familiar (18.6\%), asistencial (16.2\%), ocio o amistad (15.6\%) y otros tipos no especificados $(4.3 \%)$. Acerca de la frecuencia del contacto, un cuarto de los 
participantes manifestó tenerlo todos los días (25.2\%), seguido de varias veces por semana $(21.6 \%)$, varias veces al mes $(21.0 \%)$ y menos de una vez al mes $(10.9 \%)$. La muestra se seleccionó mediante muestreo polietápico: en primer lugar se decidieron 60 puntos de muestreo que se distribuyeron proporcionalmente a la población total de cada provincia. Posteriormente, las unidades primarias - poblaciones- se asignaron mediante muestreo aleatorio simple y las unidades últimas mediante muestreo estratificado por clusters tomando como criterio inicial la población total y como secundarios la zona (rural/urbana) y la tasa de prevalencia de cada tipo genérico de discapacidad -física, psíquica, sensorial- en la población general (i. e., personas menores de 60 años, con grado de discapacidad igual o superior al 33\%, según tipo de discapacidad principal, por municipio).

En la muestra se ha conferido mayor peso específico a las áreas sanitaria y educativa (que constituyen casi un $70 \%$ de la muestra total) atendiendo a la importancia diferencial que dichas áreas tienen para las personas con discapacidad. El trabajo de campo se realizó mediante la administración a los interesados de la Escala de Actitudes hacia las Personas con Discapacidad (cuyo proceso de construcción y validación describiremos más adelante).

\section{Instrumento}

Se ha utilizado como instrumento de base la Escala General de Actitudes hacia las Personas con Discapacidad (Arias, 1993; Arias, Verdugo y Rubio, 1995; Verdugo, Arias y Jenaro, 1994; Verdugo, Jenaro y Arias, 1995). Consiste en una escala de estimaciones sumatorias con cuatro grados de acuerdo (muy de acuerdo, bastante de acuerdo, bastante en desacuerdo, muy en desacuerdo).

El conjunto inicial de 34 ítems fue sometido a la revisión de 10 jueces, al objeto de detectar aquellos ítems que (a) resultaran claramente inadecuados para evaluar el constructo de interés; (b) fueran culturalmente inapropiados; (c) estuvieran desfasados atendiendo a la legislación española actual sobre la discapacidad; (d) estuvieran redactados de modo ambiguo; (e) se refirieran a grupos o referentes actitudinales distintos de los perseguidos por este estudio, y (f) presentaran un contenido duplicado o redundante. Además, valoraron cada uno de ellos en términos de valencia (positiva, negativa, neutra) y de idoneidad, importancia, sensibilidad y dificultad (en una escala de 1 a 4 ).

La concordancia entre los jueces fue prácticamente perfecta $(\alpha$ de Krippendorff $=$ .961) en la asignación de la valencia de los 31 ítems que configuraron la escala final, y suficientemente elevada en el resto de características consideradas. Como se puede observar en la Figura 1, el rango del coeficiente ponderado de concordancia de Bangdiwala (Bangdiwala, 1987) se situó entre $B^{W}{ }_{N}=.657$ (dificultad) y $B^{W}{ }_{N}=.931$ (importancia). 


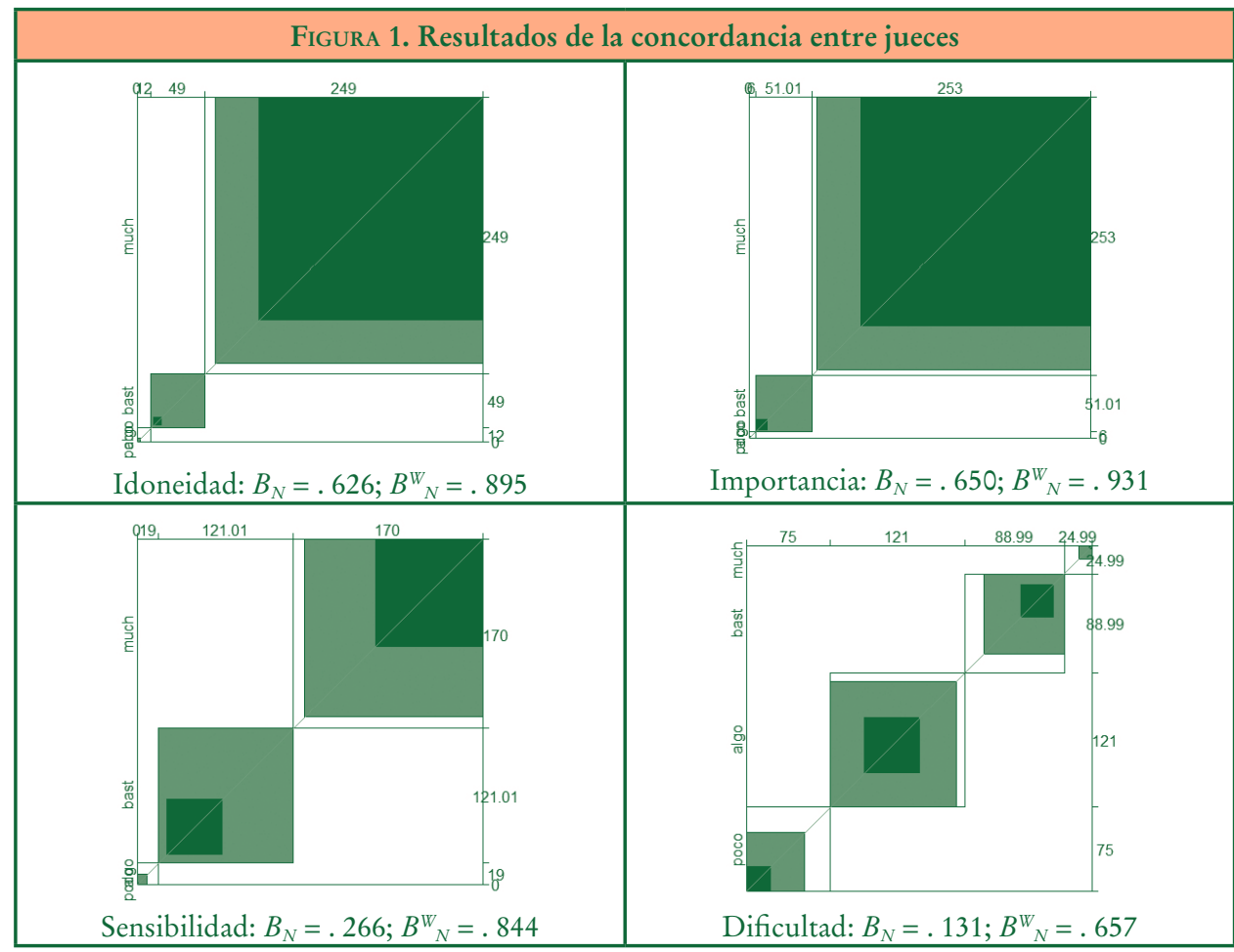

\section{Procedimiento}

Se envió a los responsables de los diferentes servicios profesionales donde se iba a aplicar la escala una comunicación en la que se les explicaban someramente los propósitos del estudio y se anunciaba la próxima visita de un encuestador. Se planificó el trabajo de campo a partir de cuatro documentos: la propia escala, el listado de profesionales a entrevistar, las normas a seguir por los entrevistadores y las acreditaciones (de la Junta de Castilla y León y de la empresa encargada del trabajo de campo).

En lo que se refiere al reclutamiento y formación de entrevistadores, se contó para la realización del trabajo de campo con entrevistadores mayores de 20 años y con un nivel mínimo de estudios correspondiente al bachillerato. Algunos de los entrevistadores contaban con experiencia contrastada en investigaciones similares. El resto fue entrenado específicamente para la presente investigación. El equipo humano estuvo compuesto por los siguientes profesionales: 1 director de campo, 2 adjuntos a la dirección de campo, 2 supervisores, 9 coordinadores de campo y 34 entrevistadores. Se puso especial empeño en la formación y entrenamiento de los entrevistadores al objeto de minimizar la varianza generada por las posibles diferencias de actuación. 
Al objeto de verificar la correcta realización del trabajo de campo, se procedió a un exhaustivo trabajo de depuración y supervisión que garantizase la calidad de la información recogida. Los controles se establecieron desde una doble vertiente: (a) verificación de que se había realizado correctamente el muestreo y (b) verificación de que el cuestionario había sido aplicado en su integridad y de manera correcta, según las instrucciones transmitidas en las sesiones formativas. Se revisaron todos los cuestionarios manualmente y, en un momento posterior, de forma automática sobre la base de la realización de comprobaciones de rango y de cobertura entre las respuestas dadas.

En una segunda fase se realizaron supervisiones de control consistentes en un chequeo telefónico del 9\% del conjunto de personas entrevistadas. En esta fase se verificó la labor de todos y cada uno de los encuestadores, así como en cada una de las 9 provincias en que se desarrolló el trabajo de campo. El chequeo telefónico sirvió asimismo para completar la labor de supervisión de los 9 coordinadores. Por último, se verificó la validez de la información recogida y grabada, controlando que la información soportada en fichero informático hubiera sido registrada correctamente. Para ello se procedió a la regrabación del 8\% de las escalas.

\section{Análisis de datos}

Tras los resultados iniciales de concordancia entre jueces a que se ha aludido en párrafos anteriores, se desarrollaron varios análisis ulteriores en tres fases.

En la fase I, se dividió la muestra original en dos submuestras aleatorias de $N=488$ participantes cada una y se calcularon las correlaciones policóricas entre los ítems. A continuación se realizó una comparación entre ambas estructuras de correlación y de varianzas/covarianzas residuales, al objeto de determinar su equivalencia (i. e., si diferían o no significativamente entre sí) utilizando ecuaciones estructurales (Jöreskog y Sörbom, 1993; Von Eye y Mun, 2005).

En la fase II, se llevó a cabo un análisis factorial exploratorio sobre la matriz de correlaciones policóricas entre los ítems (Hair, Black, Babin y Anderson, 2010) calculada sobre las respuestas de la primera mitad $(N=488)$, extraída aleatoriamente de la muestra original. Se utilizó como método de extracción el factor común con rotación oblicua PROMAX, tras la pertinente comprobación de la adecuación de los datos para el análisis $\left(K M O=.909\right.$; prueba de esfericidad de Bartlett, $\mathrm{c}^{2}=6052.888$, $p=.000$ ). Se determinó el número de factores óptimo para resumir la información contenida en los datos. Para ello se llevó a cabo un análisis paralelo optimizado (Timmerman y Lorenzo-Seva, 2011).

En la fase III, sobre la matriz de correlaciones policóricas obtenidas con la segunda submuestra aleatoria $(N=488)$ se estimaron mediante análisis factorial confirmatorio (AFC) dos modelos: unifactorial y de tres factores correlacionados. Se estimó, sobre este segundo modelo, la fiabilidad de las medidas (consistencia interna, fiabilidad de los indicadores individuales, fiabilidad de constructo y error de medida), así como la validez de contenido, convergente y discriminante. Ambos modelos se estimaron mediante mínimos cuadrados ponderados diagonalizados sobre la matriz 
de varianzas-covarianzas asintóticas, dada la ausencia de normalidad multivariada de los datos (Beauducel y Herzberg, 2006): utilizando las pruebas de Mardia, se han obtenido valores $b 1 p=63.16, p=.000 ; b 2 p=320.72, p=.000$ (para RS); $b 1 p=42.49$, $p=.000 ; b 2 p=277.8, p=.000($ para VN); y $b 1 p=24.44, p=.000 ; b 2 p=71.79, p=$ .000 (para PI). La bondad de ajuste se evaluó mediante el índice de ajuste comparativo (CFI), el índice de Tucker-Lewis (TLI) y la raíz del error cuadrático medio de aproximación (RMSEA). Para los índices CFI y TLI se estima que valores por encima de .90 y .95 indican grados de ajuste aceptables y buenos, respectivamente (Hu y Bentler, 1999). En el caso del RMSEA, valores iguales o inferiores a .05 y .08 se interpretan como buenos y aceptables, respectivamente (Browne y Cudeck, 1992; Hu y Bentler, 1999). Para establecer la relevancia de las diferencias de ajuste entre modelos, se siguieron las recomendaciones de Chen (2007) y Cheung y Rensvold (2002), según las cuales incrementos menores a .010 en CFI y TLI y decrementos menores a .015 en RMSEA sugieren que no hay cambios relevantes en el ajuste de un modelo respecto del siguiente más restrictivo. Los diferentes análisis se realizaron mediante los programas R, versión 3.2.2 (R Core Team, 2015), y LISREL, versión 9.3 (Scientific Software International, 2012).

\section{Resultados}

\section{Resultados de la fase I}

Se han sometido a prueba las hipótesis de que tanto las estructuras de correlación como las matrices de varianzas/covarianzas residuales son invariantes entre los dos grupos $\left(\Phi^{(1)}=\Phi^{(2)} ; \Theta^{(1)}=\Theta^{(2)}\right)$. Los resultados de ajuste global apoyan la verosimilitud de ambas hipótesis: $\mathrm{c}^{2}(465)=623.54, \mathrm{p}=.000 ; R M S E A=.025$ (CI 90\% $=.018-.030$; $C F I=.99 ; T L I=.99 ; S R M R=.036)$. Los resultados por grupo indicaron que ambas submuestras contribuyeron de forma muy similar al valor del $\mathrm{c}^{2}$ global, con $\mathrm{c}^{2}{ }_{(1)}=$ $323.24(51.84 \%)$ y c $^{2}{ }_{(2)}=300.30(48.16 \%)$. Asimismo, en ambas submuestras se obtuvieron valores $S R M R$ similares: $S R M R_{(1)}=.037 ; S R M R_{(2)}=.036$, y los mismos valores en el índice global de ajuste $(G F I=.96$ en ambos casos). Se han calculado, asimismo, los coeficientes de congruencia entre las saturaciones de las soluciones factoriales de ambas submuestras. Dichos coeficientes $\left(\mathrm{CC}_{\mathrm{F} 1}=.996 ; \mathrm{CC}_{\mathrm{F} 2}=.993 ; \mathrm{CC}_{\mathrm{F} 3}=.991\right)$ refrendan la equivalencia de las estructuras factoriales. En consecuencia, podemos determinar que tanto las estructuras de correlación como las varianzas y covarianzas residuales son invariantes en ambos grupos, por lo que procede realizar los análisis exploratorio y confirmatorio de las dos fases subsiguientes.

\section{Resultados de la fase II}

El análisis paralelo optimizado sobre 1000 réplicas aleatorias llegó a una solución óptima de un factor cuando se consideró el percentil 95 de la proporción de varianza 
aleatoria y de tres factores cuando se consideró la media de la misma. Este resultado sugiere la presencia de un factor fuerte y al menos dos dimensiones adicionales con menor poder explicativo. La solución de tres factores finalmente elegida explica el $62.120 \%$ de la varianza común.

En la Tabla 2 se presentan los resultados del análisis factorial. El primer factor, con un valor propio de 13.157 que explica el $42.441 \%$ de la varianza común, se ha denominado "relaciones sociales y personales con las personas con discapacidad" (en adelante, RS) y comprende 13 ítems cuyo contenido tiene que ver con sentimientos, intenciones de conducta y pensamientos de la persona cuando ha de establecer una interacción personal o social con una persona con discapacidad. El segundo factor (valor propio de 3.708 ) explica el $11.962 \%$ de la varianza común y se ha denominado "normalización" o "vida normalizada" (en adelante, VN). En él se agrupan otros 13 ítems que hacen referencia tanto a los derechos de llevar una vida normalizada y a la igualdad de oportunidades como a la posibilidad o capacidad de las personas con discapacidad para desenvolverse en distintos ámbitos de la vida como las personas sin discapacidad. El tercer factor (valor propio de 2.392) da cuenta del $7.717 \%$ de la varianza común y se refiere a "programas de intervención" (en adelante, PI) y actuaciones concretas a realizar en orden a favorecer la integración y la plena inclusión de las personas con discapacidad, así como juicios sobre la rentabilidad económica de esas actuaciones.

\begin{tabular}{|c|l|c|c|c|c|}
\hline \multicolumn{7}{|c|}{ TABLA 2. Análisis factorial exploratorio: saturaciones, comunalidades, } \\
valores propios y varianza explicada \\
\hline Factor I: Relaciones sociales y personales RS & F1 & F2 & F3 & h2 \\
\hline i07 & $\begin{array}{l}\text { En situaciones sociales, preferiría no encontrarme con } \\
\text { personas con discapacidad. }\end{array}$ & .47 & -.04 & .37 & .514 \\
\hline i09 & $\begin{array}{l}\text { En general me siento a disgusto en compañía de una } \\
\text { persona con discapacidad. }\end{array}$ & .62 & -.12 & .18 & .441 \\
\hline i10 & $\begin{array}{l}\text { Si me citara con una persona con discapacidad, me } \\
\text { preocuparía lo que la gente pudiera pensar. }\end{array}$ & .65 & -.08 & .25 & .582 \\
\hline i13 & $\begin{array}{l}\text { Si tuviera un familiar cercano con discapacidad, evitaría } \\
\text { comentarlo con otras personas. }\end{array}$ & .62 & -.06 & .25 & .550 \\
\hline i16 & $\begin{array}{l}\text { Creo que me resultaría fácil relacionarme con una } \\
\text { persona con discapacidad. }\end{array}$ & .49 & .40 & -.22 & .486 \\
\hline i18 & $\begin{array}{l}\text { Si tuviera que hablar con una persona con discapacidad, } \\
\text { me preocuparía no saber cómo comportarme. }\end{array}$ & .47 & .08 & -.04 & .251 \\
\hline i19 & $\begin{array}{l}\text { Podría citarme con una persona con discapacidad con la } \\
\text { misma facilidad que con cualquier otra persona. }\end{array}$ & .60 & .40 & -.29 & .589 \\
\hline i21 & $\begin{array}{l}\text { Me gustaría que mi hijo se relacionara con niños con } \\
\text { discapacidad. }\end{array}$ & .48 & .30 & .01 & .486 \\
\hline i24 & $\begin{array}{l}\text { Evitaría acompañar a una persona con discapacidad a } \\
\text { comer a un restaurante en el que me conocieran. }\end{array}$ & .61 & -.01 & .25 & .586 \\
\hline i25 & $\begin{array}{l}\text { El comportamiento de las personas con discapacidad es } \\
\text { irritante. }\end{array}$ & .49 & .29 & .29 & .393 \\
\hline
\end{tabular}

(C) Ediciones Universidad de Salamanca / CC BY-NC-ND

Siglo Cero, vol. 47 (2), n. ${ }^{\circ} 258,2016$, abril-junio, pp. 7-41

$$
-17-
$$




\begin{tabular}{|c|c|c|c|c|c|}
\hline \multicolumn{6}{|c|}{$\begin{array}{l}\text { TABLA 2. Análisis factorial exploratorio: saturaciones, comunalidades, } \\
\text { valores propios y varianza explicada }\end{array}$} \\
\hline \multicolumn{2}{|c|}{ Factor I: Relaciones sociales y personales RS } & F1 & F2 & $\mathrm{F} 3$ & h2 \\
\hline $\mathrm{i} 28$ & $\begin{array}{l}\text { No me importaría tener como amiga a una persona con } \\
\text { discapacidad. }\end{array}$ & .41 & .35 & .14 & .579 \\
\hline i31 & $\begin{array}{l}\text { Encontrarme con una persona con discapacidad me } \\
\text { produce tensión y malestar. }\end{array}$ & .92 & -.09 & .02 & .782 \\
\hline$i 32$ & $\begin{array}{l}\text { Si tuviera que trabajar con una persona con } \\
\text { discapacidad, me limitaría a mantener con ella una } \\
\text { relación superficial. }\end{array}$ & .68 & .06 & .12 & .608 \\
\hline \multicolumn{6}{|c|}{$\begin{array}{l}\text { Valor propio: } 13.157 \text {; Varianza común explicada: } 42.411 \% \text {; Coeficiente de congruencia: } .996 \\
\text { Coeficientes de fiabilidad: } \alpha \text { de Cronbach ordinal }=.858 ; \omega \text { de McDonald }=.949\end{array}$} \\
\hline \multicolumn{2}{|c|}{ Factor II: Normalización VN } & F1 & $\mathrm{F} 2$ & F3 & h2 \\
\hline i01 & $\begin{array}{l}\text { Las personas con discapacidad pueden sentirse tan } \\
\text { satisfechas de sí mismas como cualquier persona. }\end{array}$ & -.15 & .39 & .24 & .209 \\
\hline $\mathrm{i} 02$ & $\begin{array}{l}\text { Las personas con discapacidad deberían tener las } \\
\text { mismas oportunidades de empleo que cualquier } \\
\text { persona. }\end{array}$ & -.16 & .46 & .37 & .386 \\
\hline $\mathrm{i} 03$ & $\begin{array}{l}\text { Las personas con discapacidad son menos productivas } \\
\text { en su lugar de trabajo que las personas sin discapacidad. }\end{array}$ & .02 & .40 & .20 & .284 \\
\hline i08 & $\begin{array}{l}\text { En general, las personas con discapacidad se consideran } \\
\text { tan valiosas como cualquiera. }\end{array}$ & .01 & .46 & .40 & .537 \\
\hline i11 & $\begin{array}{l}\text { Las personas con discapacidad son capaces de adaptarse } \\
\text { a una vida independiente. }\end{array}$ & -.12 & .65 & .09 & .396 \\
\hline i14 & $\begin{array}{l}\text { Las personas con discapacidad deberían poder } \\
\text { divertirse con el resto de las personas. }\end{array}$ & .17 & .48 & .23 & .545 \\
\hline i15 & $\begin{array}{l}\text { Las personas con discapacidad pueden disfrutar del } \\
\text { deporte tanto como cualquier otra persona. }\end{array}$ & .00 & .43 & .14 & .263 \\
\hline $\mathrm{i} 20$ & $\begin{array}{l}\text { Las personas con discapacidad pueden alcanzar un } \\
\text { elevado nivel de autodeterminación. }\end{array}$ & -.04 & .81 & -.06 & .584 \\
\hline $\mathrm{i} 22$ & $\begin{array}{l}\text { Los trabajadores con discapacidad deberían recibir el } \\
\text { mismo salario que los que no tienen discapacidad. }\end{array}$ & .08 & .48 & .30 & .517 \\
\hline i23 & $\begin{array}{l}\text { Las personas con discapacidad tienen exactamente los } \\
\text { mismos derechos que el resto de las personas. }\end{array}$ & .23 & .54 & .20 & .474 \\
\hline $\mathrm{i} 27$ & $\begin{array}{l}\text { Los niños con discapacidad deberían acudir a los } \\
\text { mismos colegios que los demás niños. }\end{array}$ & .07 & .36 & .08 & .198 \\
\hline i29 & $\begin{array}{l}\text { Las personas con discapacidad pueden tener una } \\
\text { personalidad tan equilibrada como las personas sin } \\
\text { discapacidad. }\end{array}$ & .23 & .62 & -.03 & .578 \\
\hline $\mathrm{i} 30$ & $\begin{array}{l}\text { Las personas con discapacidad son tan amistosas como } \\
\text { cualquier otra persona. }\end{array}$ & .14 & .74 & .05 & .715 \\
\hline \multicolumn{6}{|c|}{$\begin{array}{l}\text { Valor propio: } 3.708 \text {; Varianza común explicada: } 11.962 \% \text {; Coeficiente de congruencia: } .993 \\
\text { Coeficientes de fiabilidad: } \alpha \text { de Cronbach ordinal }=.822 ; \omega \text { de McDonald }=.921\end{array}$} \\
\hline
\end{tabular}




\begin{tabular}{|c|c|c|c|c|c|}
\hline \multicolumn{6}{|c|}{$\begin{array}{l}\text { TABLA 2. Análisis factorial exploratorio: saturaciones, comunalidades, } \\
\text { valores propios y varianza explicada }\end{array}$} \\
\hline \multicolumn{2}{|c|}{ Factor III: Programas de intervención PI } & F1 & F2 & F3 & h2 \\
\hline i05 & $\begin{array}{l}\text { Debería hacerse algo para conseguir una mayor } \\
\text { integración de las personas con discapacidad, por } \\
\text { ejemplo, facilitándoles el acceso a los lugares públicos. }\end{array}$ & -.01 & .15 & .69 & .576 \\
\hline i06 & $\begin{array}{l}\text { Las personas con discapacidad severa deberían ser } \\
\text { confinadas en centros especiales. }\end{array}$ & .08 & -.02 & .43 & .218 \\
\hline $\mathrm{i} 26$ & $\begin{array}{l}\text { Los programas de rehabilitación para las personas con } \\
\text { discapacidad son excesivamente costosos. }\end{array}$ & .04 & .04 & .47 & .269 \\
\hline $\mathrm{i} 33$ & $\begin{array}{l}\text { Debería gastarse más dinero en suprimir las barreras } \\
\text { físicas que siguen dificultando la vida a las personas con } \\
\text { discapacidad. }\end{array}$ & .18 & .11 & .50 & .461 \\
\hline $\mathrm{i} 34$ & $\begin{array}{l}\text { El problema de los prejuicios hacia las personas con } \\
\text { discapacidad ha sido exagerado por los responsables } \\
\text { políticos y educativos. }\end{array}$ & .23 & .00 & .53 & .459 \\
\hline \multicolumn{6}{|c|}{$\begin{array}{l}\text { Valor propio: } 2.392 ; \text { Varianza común explicada: } 7.717 \% \text {; Coeficiente de congruencia }=.991 \\
\text { Coeficientes de fiabilidad: } \alpha \text { de Cronbach ordinal }=.603 ; \omega \text { de McDonald }=.802\end{array}$} \\
\hline \multicolumn{6}{|c|}{$\begin{array}{c}\text { Escala total: } \alpha \text { de Cronbach ordinal }=.928 ; \omega \text { de McDonald }=.971 \\
\alpha \text { de Cronbach estratificado }=.886\end{array}$} \\
\hline
\end{tabular}

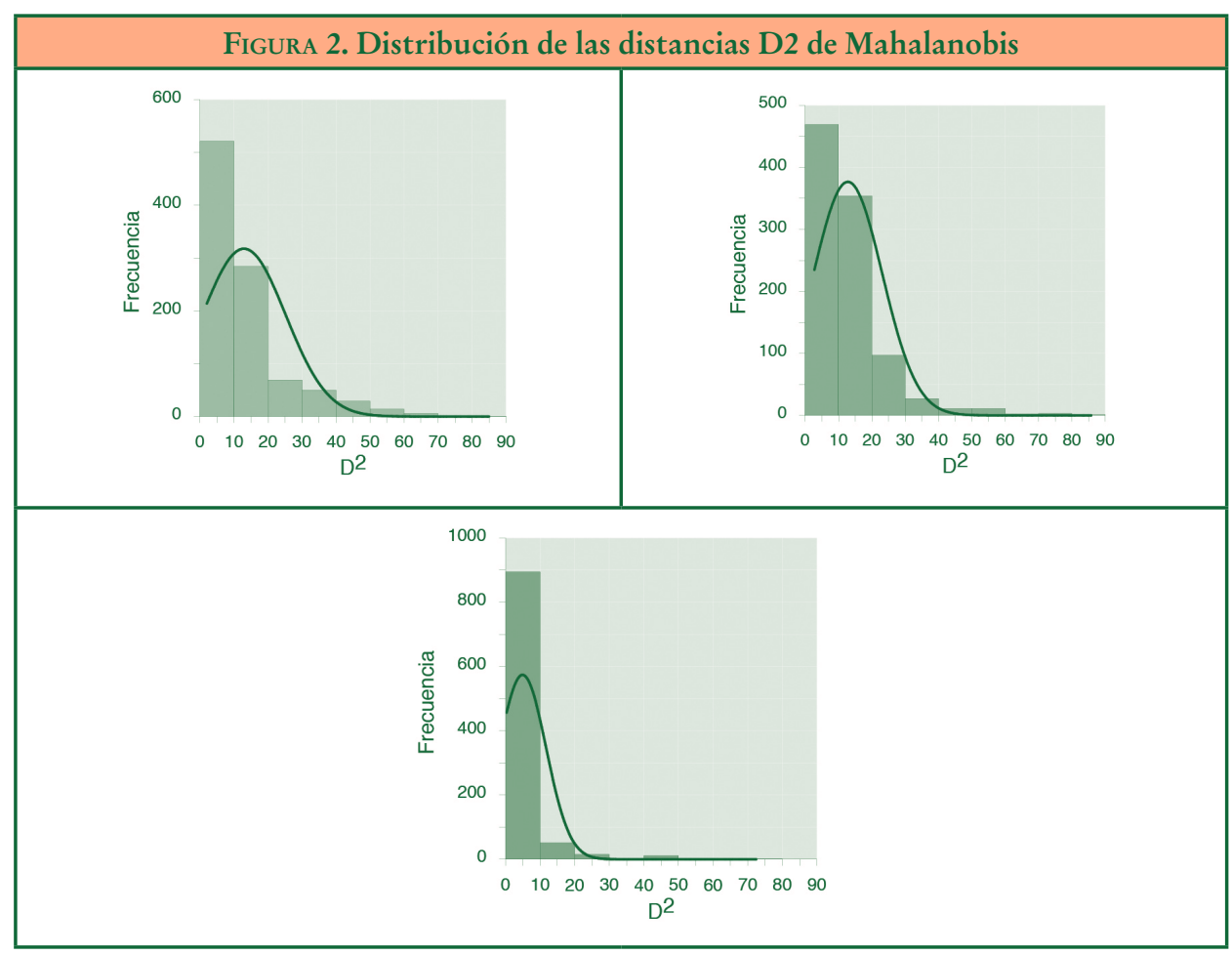

(c) Ediciones Universidad de Salamanca / CC BY-NC-ND

Siglo Cero, vol. 47 (2), n. ${ }^{\circ} 258,2016$, abril-junio, pp. 7-41

$$
-19-
$$




\section{Resultados de la fase III}

El análisis de outliers multivariados mostró que la mayor parte de las distancias $\mathrm{D}^{2}$ de Mahalanobis presentaban una clara distribución asimétrica izquierda (con el $17.47 \%$ de las distancias mayores de 20 en el factor RS; el $15.63 \%$ en el factor VN, y el $3.17 \%$ en el factor PI), por lo que no se estimó necesario eliminar ninguna observación (Figura 2), pese a la ausencia de normalidad multivariada comentada más arriba en la sección de Análisis de datos.

La comparación entre los dos modelos ha resultado muy favorable al de tres factores correlacionados. Como puede apreciarse en la Tabla 3, la mejora en el ajuste del modelo 2 sobre el 1 resultó concluyente $\left(\mathrm{Dc}^{2}(3)=843.60, p=.000 ; \triangle R M S E A=-.017\right.$, $\triangle C F I=.069, \triangle T L I=.077)$.

\begin{tabular}{|l|c|c|}
\hline \multicolumn{2}{|c|}{ TABLA 3. Bondad del ajuste de las soluciones unifactorial y de tres factores correlacionados } \\
\hline & $\begin{array}{c}\text { M1 } \\
\text { (Unifactorial) }\end{array}$ & $\begin{array}{c}\text { M2 (3 factores } \\
\text { correlacionados) }\end{array}$ \\
\hline S-Bc ${ }^{2}$ & 2229.684 & 1386.075 \\
\hline $\mathrm{GL}$ & 434 & 431 \\
\hline $\mathrm{p}$ & .000 & .000 \\
\hline $\mathrm{c}^{2} / \mathrm{GL}$ & 5.138 & 3.216 \\
\hline $\mathrm{RMSEA}$ & .065 & .048 \\
\hline (CI 90\%) & $.063-.068)$ & $.045-.051)$ \\
\hline CFI & .913 & .982 \\
\hline TLI & .907 & .984 \\
\hline SRMR & .082 & .062 \\
\hline
\end{tabular}

Nota. S-Bc ${ }^{2}=$ Satorra-Bentler chi-square; RMSEA = Root Mean Square Error of Approximation; CFI = Comparative Fit Index; TLI = Tucker-Lewis Index; SRMR = Standardized Root Mean Square Residual.

En la Tabla 4 se muestran las saturaciones, errores estándar, valores t, p y $\mathrm{R}^{2}$ de la solución estandarizada de 3 factores correlacionados. En todos los casos tanto las saturaciones como los valores de $\mathrm{R}^{2}$ han resultado estadísticamente significativos con $p<.001$.

\section{Fiabilidad}

Se entiende por fiabilidad la consistencia (i. e., reproductibilidad) de las puntuaciones a través de las réplicas de un procedimiento de evaluación (AERA, APA y NCME, 2014). Se han examinado cuatro formas de fiabilidad: consistencia interna, fiabilidad de los indicadores individuales, fiabilidad de constructo y desviación estándar (error típico de medida en el nivel manifiesto de las variables). 


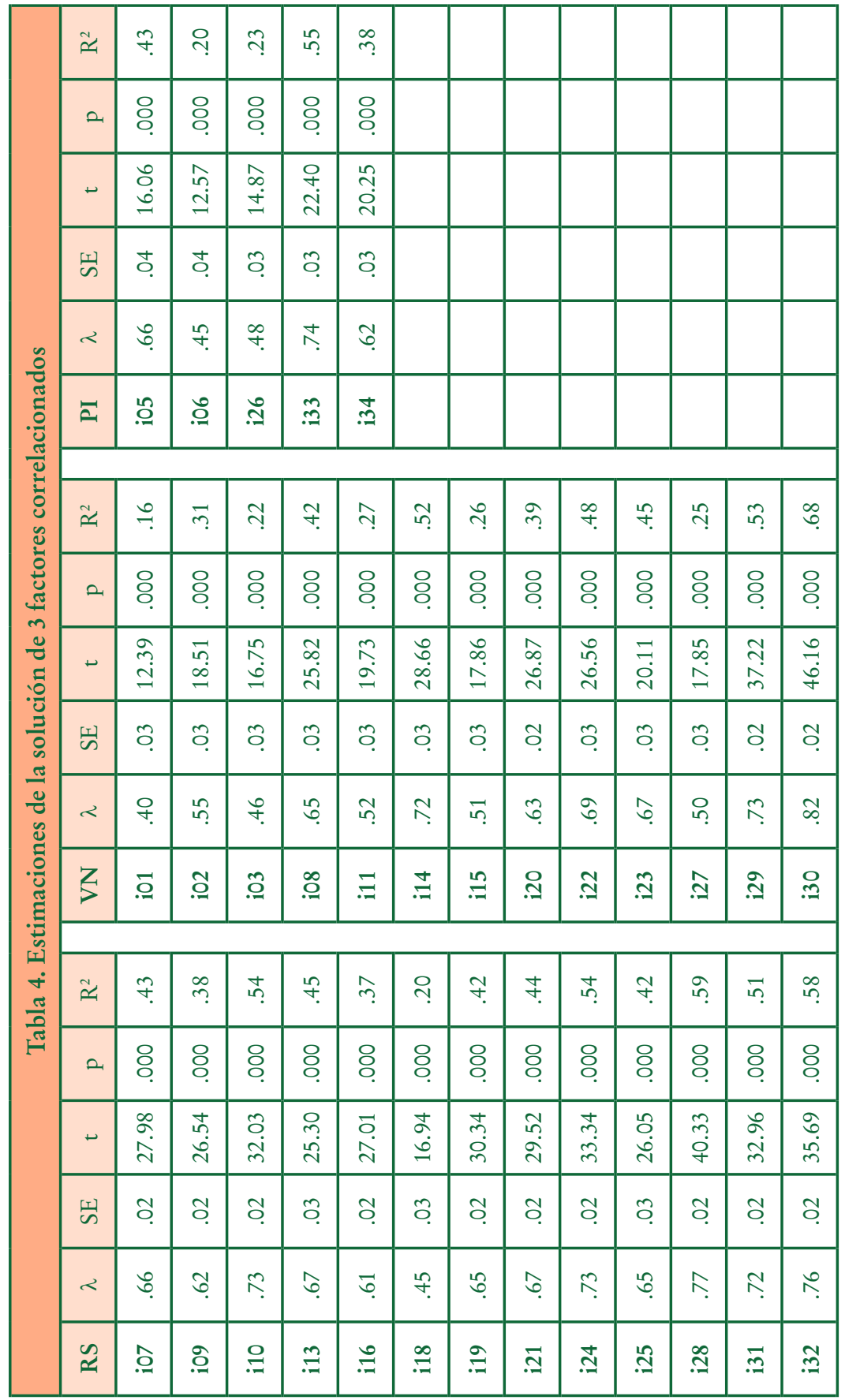

() Ediciones Universidad de Salamanca / CC BY-NC-ND

Siglo Cero, vol. 47 (2), n. ${ }^{\circ} 258,2016$, abril-junio, pp. 7-41 


\section{Consistencia interna}

Se ha calculado la consistencia interna de las tres subescalas y de la puntuación total mediante los coeficientes alfa de Cronbach, variación para datos ordinales (Gadermann, Guhn y Zumbo, 2012; Zinbarg, Revelle, Yovel y Li, 2005) y omega de McDonald (2013). Los coeficientes de consistencia interna determinan el grado en que los ítems de una subescala diseñados para medir el mismo constructo latente generan puntuaciones precisas o consistentes (Widaman, Little, Preacher y Sawalani, 2011). Además del tradicional $\alpha$ de Cronbach, se ha calculado el coeficiente $\omega$ de McDonald, con el fin de superar la asunción crítica subyacente a $\alpha$, i. e., que todos los ítems que componen cada subescala son igualmente buenos, asunción que no es aplicable en general a los ítems que componen los factores de las escalas de estimaciones sumatorias. El coeficiente $\omega$ (i. e., la razón entre la varianza verdadera y la varianza total) se ha calculado a partir de las saturaciones y las varianzas de error estimadas por el AFC (modelo de tres factores correlacionados) de acuerdo con la ecuación

$$
\omega=\frac{\sum\left(\lambda_{j}^{2}\right)}{\left[\left(\lambda_{j}^{2}\right)+\sum \theta_{j}^{2}\right]}
$$

donde $\lambda$ son las saturaciones y $\theta$ las varianzas de error, obtenidas ambas mediante AFC. Puesto que la escala se compone de tres dimensiones, se ha calculado asimismo el coeficiente $\alpha$ estratificado, dado que $\alpha$ tiende a ser subestimado cuando los coeficientes de correlación entre los factores son elevados (vid. Tabla 2).

\section{Fiabilidad de los indicadores individuales}

La fiabilidad de cada indicador puede determinarse examinando los valores $\mathrm{R}^{2}$, que muestran la cantidad de varianza de un indicador que es explicada por la variable latente (el resto de la varianza se debe al error de medida). Un valor de $\mathrm{R}^{2}$ elevado indica que el indicador es fiable. En este modelo, el indicador más fiable de RS es el ítem 28 (No me importaría tener como amiga a una persona con discapacidad) y el menos fiable el ítem 18 (Si tuviera que hablar con una persona con discapacidad, me preocuparía no saber cómo comportarme); en VN, el más fiable es el ítem 30 (Las personas con discapacidad son tan amistosas como cualquier otra persona) y el menos fiable el ítem 01 (Las personas con discapacidad pueden sentirse tan satisfechas de sí mismas como cualquier persona); en PI, el más fiable es el ítem 33 (Debería gastarse más dinero en suprimir las barreras físicas que siguen dificultando la vida a las personas con discapacidad) y el menos fiable el ítem 06 (Las personas con discapacidad severa deberian ser confinadas en centros especiales).

\section{Evidencias de frabilidad de constructo}

Además de la fiabilidad de los indicadores individuales, se ha calculado la fiabilidad compuesta de cada variable latente (fiabilidad de constructo, $r_{c}$ ). Se han obtenido 
valores de $\mathrm{r}_{\mathrm{c}}=.913(\mathrm{RS}) ; \mathrm{r}_{\mathrm{c}}=.885(\mathrm{VN}), \mathrm{y} \mathrm{r}_{\mathrm{c}}=.730(\mathrm{PI})$. Puesto que en todos los casos $r_{c}$ es superior a .70 , podemos concluir que los indicadores de las tres subescalas, considerados en conjunto, son una medida fiable del constructo. En lo que concierne a la varianza media extractada $\left(r_{v}\right)$, se han obtenido valores $r_{v}=.551(R S) ; r_{v}=.530(V N)$, y $r_{v}=.506(P I)$. Puesto que $r_{v}>.50$, podemos concluir que una cantidad sustancial de la varianza de los indicadores es capturada por el constructo comparada con la que es capturada por el error de medida. Esto proporciona confianza adicional en la operacionalización de las tres variables latentes (RS, VN, PI) que componen la escala.

\section{Error de medida}

El uso de modelos de ecuaciones estructurales para normalizar las puntuaciones de las escalas es beneficioso porque permite la estimación de parámetros fiables que toman en consideración el error de medida (Seo, Little, Shogren y Lang, en prensa).

$$
S E M T=S D \sqrt{1-\omega}
$$

De acuerdo con la ecuación (2), donde $S D=$ desviación estándar y $\omega=$ coeficiente omega de fiabilidad, los errores típicos de medida de las puntuaciones manifiestas han sido aceptables: 1.028 para RS; 1.226 para VN; 0.927 para PI; y 1.570 para la puntuación total.

\section{Evidencias de validez}

La validez se refiere al grado en que la evidencia y la teoría apoyan las interpretaciones de las puntuaciones de las pruebas de acuerdo con los usos que se proponen (AERA et al., 2014). La validez no es un concepto absoluto y, además, puede adoptar formas diversas en función del propósito con el que se interpretan los resultados. Examinaremos sucintamente distintas evidencias de validez de la escala de actitudes: de contenido, convergente y discriminante.

\section{Evidencias de validez de contenido}

La validez de contenido se define como el grado en que una prueba mide con precisión una muestra representativa de las conductas de interés (Taylor, 2008). En la construcción de la presente escala de actitudes se ha procurado garantizar la validez de contenido mediante (a) la revisión sistemática de la literatura en torno a la evaluación de actitudes hacia la discapacidad; (b) la selección de los ítems de forma que cubrieran los tres dominios teóricos de la actitud, y (c) la evaluación por medio de jueces expertos de la idoneidad, la importancia, la sensibilidad, la dificultad y la valencia de los ítems (los resultados de este último análisis se han sintetizado en párrafos precedentes). 


\section{Evidencias de validez convergente}

Existen evidencias de validez convergente si los ítems que son indicadores de un constructo específico comparten una alta proporción de la varianza común. Esto queda refrendado en el presente caso, toda vez que (a) las saturaciones de todos los ítems han resultado estadísticamente significativas; (b) con excepción de cinco, todas ellas han sido superiores a .5; y (c) la varianza media extraída de las saturaciones de los ítems en cada factor puede asimismo considerarse como un indicador de la convergencia.

\section{Evidencias de validez discriminante}

La validez discriminante proporciona evidencia de que cada constructo evaluado es único (y, por tanto, diferente de otros constructos). Se ha determinado la validez discriminante siguiendo tres acercamientos (Hair et al., 2010). En primer lugar, se ha fijado a 1 la correlación entre cada par de factores (RS-VN; RS-PI; VN-PI) y se ha comparado el ajuste de los modelos resultantes con el ajuste del modelo original de 3 factores correlacionados. Los resultados han evidenciado que este modelo es significativamente superior a los modelos en los que se ha fijado a 1 la correlación entre RS y $\mathrm{VN}\left(\mathrm{Dc}^{2}(1)=19.50, p=.000\right)$; entre RS y PI $\left(\mathrm{Dc}^{2}(1)=46.30, p=.000\right)$; y entre VN y PI $\left(\operatorname{Dc}^{2}(1)=13.34, p=.000\right)$. En segundo lugar, los valores AVE (Fornell y Larcker, 1981) han resultado superiores a los cuadrados de las correlaciones entre los factores $\left(r_{R S-V N}^{2}=.442 ; r_{R S-P I}^{2}=.144 ; r_{V N-P I}^{2}=.158\right)$, lo que indica que cada factor explica más varianza de las medidas de los ítems que lo componen que la que comparte con el resto de los factores. En tercer lugar, se ha constatado la ausencia de saturaciones cruzadas, como puede observarse en la Tabla 2.

\begin{tabular}{|l|c|c|c|}
\hline \multicolumn{4}{|c|}{ TABLA 5. Intercorrelaciones latentes y (manifiestas) entre los factores } \\
(solución de 3 factores correlacionados)
\end{tabular}

En la Tabla 5 se muestran las correlaciones entre los factores, tanto latentes como manifestas. Estas últimas son menores debido al error de medida ajustado. Todas ellas han resultado significativas con $\mathrm{p}<.001$.

\section{Discusión y conclusiones}

El principal objetivo planteado en el presente estudio se centró en actualizar y validar (a partir de versiones existentes) una escala de actitudes hacia las personas con 
discapacidad dirigida preferentemente a profesionales que están en contacto más o menos frecuente con estas personas. Tal objetivo se centraba en la necesidad de disponer de instrumentos psicométricamente sólidos de evaluación de las actitudes hacia el mencionado colectivo, a la vista de la práctica inexistencia en nuestro país de escalas de evaluación desarrolladas con rigor y garantías métricas suficientes. Estimamos que tal objetivo se ha cumplido, toda vez que (a) el procedimiento de muestreo ha garantizado trabajar con una muestra amplia y representativa de la población de referencia y (b) las cualidades métricas de validez y fiabilidad en sus diversas variantes han satisfecho los criterios mínimos de calidad exigibles a un instrumento de esta naturaleza.

En lo tocante a la fiabilidad, el instrumento ha demostrado que las tres subescalas que lo componen presentan adecuada consistencia interna, suficiente fiabilidad de los indicadores individuales, una fiabilidad de constructo adecuada y un error típico de medida relativamente bajo. Respecto a las evidencias de validez, se ha garantizado la validez de contenido mediante un examen exhaustivo de decenas de escalas de evaluación de actitudes hacia la discapacidad publicadas en otros países, y se ha obtenido una concordancia entre jueces externos entre razonable y elevada en diferentes aspectos inherentes a los ítems tales como importancia, idoneidad, dificultad y sensibilidad. Han resultado algo más cuestionables las evidencias de validez convergente (ya que en cinco de los ítems no se ha alcanzado el umbral de .5) y las de validez discriminante: los resultados del análisis paralelo apuntan a la existencia de un factor común fuerte, lo que queda refrendado por los elevados valores de las correlaciones entre las tres variables latentes (relaciones sociales y personales, vida normalizada, programas de intervención). Estos resultados supondrían la necesidad de continuar investigando sobre la dimensionalidad de las actitudes hacia las personas con discapacidad tal como se propone desde el instrumento desarrollado y tratar de clarificar si modelos alternativos al de tres factores correlacionados que aquí se ha contrastado, por ejemplo, modelos ESEM (Exploratory Structural Equation Modeling) o bifactor ESEM (Asparouhov y Muthén, 2009; Brunner, Nagy y Wilhelm, 2012; Jennrich y Bentler, 2011, 2012; Reise, 2012; Reise, Scheines, Widaman y Haviland, 2013), explican o reproducen con más precisión la variabilidad de los datos.

Fuera de los propósitos de la presente investigación ha quedado el estudio de las posibles relaciones entre las puntuaciones obtenidas en la escala y diversas variables sociodemográficas tales como la edad, el género, el nivel de formación, la especialidad, o el tipo, cantidad, razón y calidad del contacto de los profesionales con personas con discapacidad. Algunas de esas variables han sido analizadas en estudios clásicos sobre este problema, llegando en muchas ocasiones a resultados inconsistentes, por lo que parece aconsejable seguir investigando en esa vía y clarificar en lo posible la naturaleza y magnitud de tales relaciones.

Como ya se ha mencionado, la escala que presentamos ha sido validada con profesionales y, en consecuencia, los baremos que se ofrecen en el Apéndice B servirían, en principio, para calcular las puntuaciones derivadas cuando el instrumento se administre a profesionales en relación con la discapacidad. Sería conveniente comprobar si tales baremos presentan diferencias con los que se construyeran a partir de puntuaciones obtenidas de una muestra de la población general. La investigación señala 
repetidamente la necesidad de llevar a cabo estudios de calidad contrastada sobre las actitudes de la población general hacia la discapacidad, especialmente en lo que tiene que ver con el grado de conocimiento de la discapacidad y con las creencias causales (Scior, 2011). Naturalmente, de los resultados de esos estudios deberían emerger actuaciones dirigidas a mejorar el conocimiento, aumentar el realismo de las creencias y modificar, en su caso, las actitudes negativas (Rello, Garoz y Díaz, 2014).

\section{Referencias bibliográficas}

Akrami, N., Ekehammer, B., Claesson, M. y Sonnander, K. (2006). Classical and modern prejudice: Attitudes toward people with intellectual disabilities. Research in Developmental Disabilities, 27, 605-617.

Ali, A., Strydom, A., Hassiotis, A., Williams, R. y King, M. (2008). A measure of perceived stigma in people with intellectual disability. British Journal of Psychiatry, 193 (5), 410-415.

American Educational Research Association (aera), American Psychological Association (apa) y National Council on Measurement in Education (ncme) (2014). Standards for educational and psychological testing. Washington, DC: AERA.

AnTONAK, R. F. (1979). An orderic-theoretic analysis of attitudes toward disabled persons. Rebabilitation Psychology, 26, 136-144.

Antonak, R. F. (1981). Development and psychometric analysis of the Scale of Attitudes Toward Disabled Persons (Technical Report n. ${ }^{\circ}$ 1). Durham, NH: University of New Hampshire, Education Department.

AntonaK, R. F. (1985). Construct validation of the Scale of Attitudes toward Disabled Persons. The Journal of Applied Rehabilitation Counseling, 16 (1), 7-10.

AnTONAK, R. F. y LivneH, H. (1994). Direct and indirect methods to measure attitudes toward persons with disabilities, with an exegesis of the error-choice test method. Rebabilitation Psychology, 40, 3-24.

Antonak, R. F. y Livneh, H. (2000). Measurement of attitudes towards persons with disabilities. Disability and Rehabilitation: An International Multidisciplinary Journal, 22, 211-224.

AntonaK, R. F. y Rankin, P. R. (1982). Measurement and analysis of knowledge and attitudes about epilepsy and persons with epilepsy. Social Science and Medicine, 16, 1591-1593.

ARIAS, B. (1993). Evaluación de actitudes hacia la integración de alumnos con necesidades educativas especiales (Tesis doctoral no publicada). Salamanca: Universidad de Salamanca.

Arias, B. y Morentin, R. (2004). Opiniones y actitudes de las familias de personas con discapacidad en Castilla y León. Valladolid: Junta de Castilla y León.

Arias, B., Verdugo, M. Á. y Rubio, V. J. (1995). Evaluación de la Actividad Modelo Local de Valladolid. Madrid: Ministerio de Educación y Ciencia.

Asparouhov, T. y Muthén, B. (2009). Exploratory structural equation modeling. Structural Equation Modeling, 16, 397-438. doi:10.1080/10705510903008204.

Bagley, M. T. y Greene, J. F. (1981). Peer attitudes toward the handicapped. Austin, TX: Pro-Ed.

Bailey, A., Barr, O. y Bunting, B. (2001). Police attitudes toward people with intellectual disability: An evaluation of awareness training. Journal of Intellectual Disability Research, 45, 344-350. doi:10.1046/j.1365-2788.2001.00339.x.

BANGDIWALA, K. (1987). Using SAS software graphical procedures for the observer agreement chart. Proceedings of the SAS User's Group International Conference, 12, 1083-1088. 
EVALUACIÓN DE ACTITUDES DE LOS PROFESIONALES HACIA LAS PERSONAS CON DISCAPACIDAD

V. ARIAS GONZÁLEZ, B. ARIAS MARTÍNEZ, M. Á. VERDUGO ALONSO, M. RUBIA AVI Y C. JENARO RÍO

Bartlett, C. J., Quay, L. C. y Wrightsman, L. S. (1960). A comparison of two methods of attitude measurement: Likert-type and forced choice. Educational and Psychological Measurement, 20, 699-704.

Beauducel, A. y Herzberg, P. Y. (2006). On the performance of maximum likelihood versus means and variance adjusted weighted least squares estimation in CFA. Structural Equation Modeling, 13 (2), 186-203. doi:10.1207/s15328007sem1302_2.

Berryman, J. D. y Neal, W. R. (1980). The cross validation of the Attitudes Toward Mainstreaming Scale. Educational and Psychological Measurement, 40, 469-474.

Bigby, C., Clement, T., Mansell, J. y Beadle-Brown, J. (2009). 'It's pretty hard with our ones, they can't talk, the more able bodied can participate’: Staff attitudes about the applicability of disability policies to people with severe and profound intellectual disabilities. Journal of Intellectual Disability Research, 53, 363-376. doi:10.1111/j.1365-2788.2009.01154.x.

Bohner, G. y Wänke, M. (2014). Attitudes and Attitude Change. Philadelphia, PA: Psychology Press.

Breckler, S. J. y Wiggins, E. C. (1992). On defining attitude and attitude theory: Once more with feeling. En A. R. Pratkanis, S. J. Breckler y A. C. Greenwald (Eds.), Attitude structure and function (pp. 407-427). Hillsdale, NJ: Erlbaum.

Browne, M. W. y Cudeck, R. (1992). Alternative ways of assessing model fit. Sociological Methods E Research, 21, 230-258. doi:10.1177/0049124192021002005.

Brunner, M., Nagy, G. y Wilhelm, O. (2012). A tutorial on hierarchically structured constructs. Journal of Personality, 80 (4), 796-846. doi:10.1080/10107013051070418.

Campbell, J. M. (2008). Brief report: reliability and validity of the Shared Activities Questionnaire as a measure of middle school students' attitudes toward autism. Journal of Autism Developmental Disorders, 38, 1598-1604.

Canimas, J. (2015). ¿Discapacidad o diversidad funcional? Siglo Cero, 46 (2), 79-97. doi: http:// dx.doi.org/10.14201/scero20154627997.

Chadd, E. H. y Pangilinan, P. H. (2011). Disability attitudes in health care: a new scale instrument. American Journal of Physical Medicine Rebabilitation, 90 (1), 47-58.

Chen, F. F. (2007). Sensitivity of goodness of fit indices to lack of measurement invariance. Structural Equation Modeling, 14, 464-504. doi:10.1080/10705510701301834.

Cheung, G. W. y Rensvold, R. B. (2002). Evaluating goodness-of-fit indexes for testing measurement invariance. Structural Equation Modeling, 9, 233-255. doi:10.1207/S15328007SEM0902_5.

Clayfield, J. C., Fletcher, K. E. y Grudzinkas, A. J. (2011). Development and validation of the mental health attitude survey for police. Community Mental Health, 47 (6), 742-751.

Cohen, J. S. y Struening, E. L. (1959). Factors underlying opinions about mental illness in the personnel of a large mental hospital. American Psychologist, 14, 339.

Cohen, J. S. y Struening, E. L. (1960). Attitudes toward the mental ill of psychiatric hospital personnel as a function of occupation, education, age and sex. American Psychologist, 15, 417.

Cohen, J. S. y Struening, E. L. (1962). Opinions about mental illness in the personnel of two large mental hospitals. Journal of Abnormal and Social Psychology, 64, 349-360.

Cooper, A., Rose, J. y Mason, O. (2004). Measuring the attitudes of human service professionals toward deafness. American Annals of Deafness, 148 (5), 385-389.

Cowen, E. L., Bobrove, P. H., Rockway, A. M. y Stevenson, J. (1967). Development and evaluation of an attitude to deafness scale. Journal of Personality and Social Psychology, 6 (2), 297-304.

Cowen, E. L., Underberg, R. P. y Verillo, R. T. (1958). The development and testing of an attitude to blindness scale. The Journal of Social Psychology, 48, 297-304.

(C) Ediciones Universidad de Salamanca / CC BY-NC-ND

Siglo Cero, vol. 47 (2), n. ${ }^{\circ}$ 258, 2016, abril-junio, pp. 7-41 
EVALUACIÓN DE ACTITUDES DE LOS PROFESIONALES HACIA LAS PERSONAS CON DISCAPACIDAD

V. ARIAS GONZÁLEZ, B. ARIAS MARTÍNEZ, M. Á. VERDUGO ALONSO, M. RUBIA AVI Y C. JENARO RÍO

Eagly, A. y Chaiken, S. (1995). Attitude strength, attitude structure and resistance to change. En R. PetTy and J. Kosnik (Eds.), Attitude Strength (pp. 413-432). Mahwah, NJ: Erlbaum.

Edwards, N., Lennox, N. y White, P. (2007). Queensland psychiatrists' attitudes and perceptions of adults with intellectual disability. Journal of Intellectual Disability Research, 51, 75-81. doi:10.1111/j.1365-2788.2006.00870.x.

Efron, R. E. y Efron, H. Y. (1967). Measurement of attitudes toward the retarded and an application with educators. American Journal of Mental Deficiency, 72, 100-106.

Esposito, B. G. y PeACH, W. J. (1983). Changing attitudes of preschool children toward handicapped persons. Exceptional Children, 49 (4), 361-363.

Fellinghauer, B., Roth, A., Bugari, K. y Reinhardt, J. (2011). Construct validity, test-retest reliability, and internal consistency of the Photo Elicitation Semantic Differential scale (PESD) in disability studies. Journal of Developmental Physical Disabilies, 23, 257-265.

FiedleR, C. y Simpson, R. (1987). Modifyint the attitudes of non-handicapped high school students toward handicapped peers. Exceptional Children, 19, 342-349.

Findler, L., Vilchinsky, N. y WeRner, S. (2007). The multidimensional attitudes scale toward persons with disabilities (MAS): construction and validation. Rebabilitation Counseling Bulletin, 50 (3), 166-176.

Forgas, J. P., Cooper, J. y Crano, W. D. (2010). The Psychology of Attitudes and Attitude Change. New York: Psychology Press.

Fornell, C. y LARCKER, D. F. (1981). Evaluating structural equation models with unobservable variables and measurement error. Journal of Marketing Research, 18 (1), 39-50.

GadermanN, A. M., GuHN, M. y Zumbo, B. D. (2012). Estimating ordinal reliability for Likerttype and ordinal item response data: A conceptual, empirical, and practical guide. Practical Assessment, Research E Evaluation, 17 (3), 1-13.

Gan, J., Tymchuk, A. J. y Nishinara, A. (1977). Mildly retarded adults: their attitudes toward retardation. Mental Retardation, 15, 5-9.

Gilbert, D. C. y Levinson, D. J. (1956). Ideology, personality, and institutional policy in the mental hospital. The Journal of Abnormal and Social Psychology, 53 (3), 263-271.

Graham, S., Herbert, R., Price, S. y Williams, S. (2004). Attitudes and emotions of trainees in learning disability psychiatry. Psychiatric Bulletin, 28, 254-256. doi:10.1192/pb.28.7.254.

Grand, S. A., Bernier, J. E. y Strohmer, D. C. (1982). Attitudes toward disabled persons as a function of social context and specific disability. Rehabilitation Psychology, 27, 165-174.

Groomes, D. y Linkowski, D. (2007). Examining the structure of the revised acceptance disability scale. Journal of Rehabilitation, 73 (3), 3-9.

Hair, J. F., Black, W. C., Babin, B. J. y Anderson, R. E. (2010). Multivariate Data Analysis, 7th. ed. Upper Saddle River, NJ: Prentice Hall.

Hampton, N. y XiaO, F. (2007). Attitudes toward people with developmental disabilities in Chinese and American students: The role of cultural values, contact, and knowledges. The Journal of Rebabilitation, 73 (4). Retrieved from http://findarticles.com/p/articles/ mi_m0825/is_4_73/ai_n24354968/?tag=content;col1.

Hampton, N. y XiAO, F. (2008). Psychometric properties of the mental retardation attitude Inventory-revised in Chinese college students. Journal of Intellectual Disability Research, 52 (4), 299-308.

Harth, R. (1974). Attitudes and mental retardation. A review of literature. Training School Bulletin, 69, 150-164.

Hatton, C., Akram, Y., Robertson, J., Shah, R. y Emerson, E. (2003). The disclosure process and its impact on South Asian families with a child with severe intellectual disabilities. Journal of Applied Research in Intellectual Disabilities, 16 (3), 177-188.

(C) Ediciones Universidad de Salamanca / CC BY-NC-ND

Siglo Cero, vol. 47 (2), n. ${ }^{\circ}$ 258, 2016, abril-junio, pp. 7-41 
EVALUACIÓN DE ACTITUDES DE LOS PROFESIONALES HACIA LAS PERSONAS CON DISCAPACIDAD

V. ARIAS GONZÁLEZ, B. ARIAS MARTÍNEZ, M. Á. VERDUGO ALONSO, M. RUBIA AVI Y C. JENARO RÍO

Henry, D., Keys, C., Jopp, F. y Balcazar, F. (1996). The Community Living Attitudes Scale, mental retardation form: development and psychometric properties. Mental Retardation, 34 (3), 149-158.

Horne, M. D. (1985). Attitudes toward handicapped students: Professional, peer, and parent reactions. Hillsdale, NJ: Lawrence Erlbaum Associates.

Hu, L. y Bentler, P. M. (1999). Cutoff criteria for fit indexes in covariance structure analysis: Conventional criteria versus new alternatives. Structural Equation Modeling, 6, 1-55. doi:10.1080/10705519909540118.

Jennitich, R. I. y Bentler, P. M. (2011). Exploratory bi-factor analysis. Psychometrika, 76, 537-549. doi:10.1007/s11336-011-9218-4.

Jennitich, R. I. y Bentler, P. M. (2012). Exploratory bi-factor analysis: The oblique case. Psychometrika, 77, 442-454. doi:10.1007/s11336-012-9269-1.

Jöreskog, K. G. y Sörbom, D. (1993). LISREL user's reference guide. Chicago, IL: Scientific Software International.

Kassam, A., Glozier, N., Leese, M., Henderson, C. y Thomicroft, G. (2010). Development and responsiveness of a scale to measure clinicians' attitudes to people with mental illness (medical student version). Acta Psychiatrica Scandinavica, 122 (2), 153-161.

Kiertsch, M. S., Hanson, P. L. y DuHoux, M. A. (1980). Preparing the mainstream: Changing children's attitudes toward the disabled. School Psychology Review, 9 (3), 279-283.

Langevin, M. y Hagler, P. (2004). Development of a scale to measure peer attitudes toward children who stutter. En A. BOTHE (Ed.), Evidence-based Treatment of Stuttering: Empirical Bases and Clinical Applications. New Jersey: Lawrence Erlbaum Associates.

LarriveE, B. y CoOK, L. (1979). Mainstreaming: A study of the variables affecting teacher attitudes. Journal of Special Education, 13 (3), 315-324.

Lazar, A. L., Gensley, J. T. y Orpet, R. E. (1971). Changing attitudes of young gifted children toward handicapped persons. Exceptional Children, 37, 600-602.

LidSLEy, J. D. y FriTh, G. H. (1983). The effects of nonhandicapped students' personal characteristics on their attitude toward handicapped peers. Journal for Special Educators, 20, 64-69.

Lilienfeld, M. y Alant, E. (2002). Attitudes of children toward an unfamiliar peer using an AAC device with and without voice output. Angmentative Alternative Communication, $18,91-101$.

LivneH, H. (1985). Factor structure of attitudes toward individuals with disabilities: A replication. Rebabilitation Counseling Bulletin, 29, 53-58.

LOMBARdi, A. R. y Murray, C. (2011). Measuring university faculty attitudes toward disability: Willingness to accommodate and adopt Universal Design principles. Journal of Vocational Rehabilitation, 34, 43-56. doi:10.3233/JVR-2010-0533.

Marsh, V. y Friedman, R. (1972). Changing public attitudes toward blindness. Exceptional Children, 38, 426-428.

McDonald, R. P. (2013). Test theory: A unified treatment. New York: Psychology Press.

Miller, M., Armstrong, S. y Hagan, M. (1981). Effects on teaching on elementary students' attitudes toward handicapped. Education and Training of the Mentally Retarded, 16, 110-113.

Morin, D., Crocker, G., Beaulieu-Bergeron, R. y Caron, J. (2013). Validation of the attitudes toward intellectual disability e ATTID questionnaire. Journal of Intellectual Disability Research, 57 (3), 268-278.

Nunnally, J. C. (1961). Popular conceptions of mental health: their development and change. New York: Hol, Rinehart and Winston.

OstapCZuK, M. y Musch, J. (2011). Estimating the prevalence of negative attitudes towards people with disability: A comparison of direct questioning, projective questioning and

(C) Ediciones Universidad de Salamanca / CC BY-NC-ND

Siglo Cero, vol. 47 (2), n. ${ }^{\circ}$ 258, 2016, abril-junio, pp. 7-41 
randomised response. Disability and Rehabilitation: An International, Multidisciplinary Journal, 33, 399-411. doi:10.3109/09638288.2010.492067.

Parish, T. S. y Copeland, T. F. (1978). Teachers' and students' attitudes in mainstreamed classrooms. Psychological Reports, 43 (1), 54.

Popovich, P. M., Scherbaum, C. A., Scherbaum, K. L. y Polinko, N. (2003). The assessment of attitudes toward individuals with disabilities in the workplace. Journal of Psychology, 137 (2), 163-177.

Power, M. J., Green, A. M., CAtchpole, C. et al. (2010). The Attitudes to Disability Scale (ADS): development and psychometric properties. Journal of Intellectual Disability, 54 (9), 860-874.

Pruett, S. y Chan, F. (2006). Development and psychometric validation of the disability attitude implicit association test. Rebabilitation Psychology, 51 (3), 202-213.

R Core Team (2015). R: A language and environment for statistical computing. Vienna, Austria: R Foundation for Statistical Computing. URL: http://www.R-project.org/.

ReISE, S. P. (2012). The rediscovery of bifactor measurement models. Multivariate Behavioral Research, 47 (5), 667-696. doi:10.1080/00273171.2012.715555.

Reise, S. P., Scheines, R., Widaman, K. y Haviland, M. (2013). Multidimensionality and structural coefficient bias in structural equation modeling: a bifactor perspective. Educational and Psychological Measurement, 73 (1), 5-26.

Rello, C. F., Garoz, I. y Díaz, M. (2014). Determinantes de las técnicas de cambio de actitudes hacia la discapacidad en programas educativos: una revisión internacional. Siglo Cero, 45 (3), 28-46.

Reynolds, W. M. y Greco, V. T. (1980). The reliability and factorial validity of a scale for measuring teachers' attitudes toward mainstreaming. Educational and Psychological Measurement, 40, 463-468.

Rosenbaum, P., Armstrong, R. y King, S. (1985). Children's attitudes toward disabled peers: a self-report measure. Journal of Pediatric Psychology, 11 (4), 517-530.

Rucker, C. y Gable, R. (1974). The Rucker-Gable Educational Programming Scale. Storrs, CT: Rucker-GABLE Associates.

Saunders, G. H., Cienkowski, K. M., Forsline, A. y Fausti, S. (2005). Normative data for the attitudes towards loss of hearing questionnaire. Journal of the American Academy of Audiology, 16 (9), 637-652.

Schmelkin, L. P. (1981). Teachers' and non-teachers' attitudes toward mainstreaming. Exceptional Children, 48, 42-47.

Scientific Software International (2013). Lisrel (Version 9.3) [Computer software]. Lincolnwood, IL: Scientific Software International.

Scior, K. (2011). Public awareness, attitudes and beliefs regarding intellectual disability: A systematic review. Research in Developmental Disabilities, 32 (6), 2164-2182.

Scior, K. y Furnham, A. (2011). Development and validation of the Intellectual Disability Literacy Scale for assessment of knowledge, beliefs and attitudes to intellectual disability. Research in Developmental Disabilities, 32, 1530-1541. doi:10.1016/j.ridd.2011.01.044.

SCIOR, K. y Werner, S. (2015). Changing attitudes to learning disability: A review of the evidence. Jerusalem: Mencap.

Seo, H., Little, T. D., Shogren, K. A. y Lang, K. M. (in press). On the benefits of latent variable modeling for norming scales: The case of the Supports Intensity Scale - Children's version. International Journal of Behavioral Development. doi:10.1177/0165025415591230.

Siller, J., Ferguson, L. T., Vann, D. H. y Holland, B. (1967). Structure of attitudes toward the physically disabled: The Disability Factor Scales - Amputation, Blindness, Cosmetic

(C) Ediciones Universidad de Salamanca / CC BY-NC-ND

Siglo Cero, vol. 47 (2), n. ${ }^{\circ}$ 258, 2016, abril-junio, pp. 7-41 
EVALUACIÓN DE ACTITUDES DE LOS PROFESIONALES HACIA LAS PERSONAS CON DISCAPACIDAD

V. ARIAS GONZÁLEZ, B. ARIAS MARTÍNEZ, M. Á. VERDUGO ALONSO, M. RUBIA AVI Y C. JENARO RÍO

Conditions. Studies in Reaction to Disability: XII. New York: New York University School of Education.

Symons, A. B., Fish, R., McGuigan, D., Fox, J. y Akt, E. A. (2012). Development of an instrument to measure medical students' attitudes toward persons with disabilities. Intellectual and Developmental Disabilies, 50 (3), 251-260.

Tachibana, T. y WatAnabe, K. (2003). Schemata and attitude toward persons with intellectual disability in Japan. Psychological Reports, 93, 1161-1172.

Tachibana, T. y Watanabe, K. (2004). Attitudes of Japanese adults toward persons with intellectual disability: Relationship between attitudes and demographic variables. Education and Training in Developmental Disabilities, 39, 109-126.

TAYLOR, R. L. (2008). Assessment of exceptional students: Educational and psychological procedures (8th ed.). Upper Saddle River, NJ: Pearson.

TAYlor, S. y DeAR, M. (1981). Scaling community attitudes toward the mentally ill. Schizophrenia Bulletin, 7 (2), 225-240.

Thomas, A., Palmer, J., Coker-Juneau, C. y Williams, D. (2002). Factor structure and construct validity of the interaction with disabled persons scale. Educational and Psychological Measurement, 63 (3), 465-483.

Timmerman, M. E. y Lorenzo-Seva, U. (2011). Dimensionality assessment of ordered polytomous items with parallel analysis. Psychological Methods, 16 (2), 209.

Towfighy-Hoshyar, N. y Zingle, H. W. (1984). Regular class students' attitudes toward integrated multiply handicapped peers. American Journal of Mental Deficiency, 88 (6), 630-637.

Triandis, H. C. (1971). Attitudes and attitude change. New York: John Wiley and Sons.

TRINGO, J. L. (1970). The hierarchy of preferences toward disability groups. Journal of Special Education, 4, 295-306.

UPton, T. D. y HARPER, D. C. (2002). Multidimensional disability attitudes and equitable evaluation of educational accommodations in higher education. Journal of Postsecondary Education and Disability, 15 (2), 115-130.

Verdugo, M. Á., Arias, B. y Jenaro, C. (1994). Actitudes hacia las personas con minusvalía. Madrid: Instituto Nacional de Servicios Sociales.

Verdugo, M. Á., Jenaro, C. y Arias, B. (1995). Actitudes sociales y profesionales hacia las personas con discapacidad: estrategias de evaluación e intervención. En M. Á. Verdugo (Dir.), Personas con discapacidad: perspectivas psicopedagógicas y rehabilitadoras (pp. 79143). México: Siglo XXI.

Voeltz, L. M. (1980). Effects of structured interactions with severely handicapped peers to children's attitudes. American Journal of Mental Deficiency, 86 (4), 380-390.

Von Eye, A. y Mun, E. Y. (2005). Analyzing rater agreement: Manifest variable methods. Mahwah, NJ: Lawrence Erlbaum Associates.

Widaman, K. F., Little, T. D., Preacher, K. J. y Sawalani, G. M. (2011). On creating and using short forms of scales in secondary research. En K. H. TRzesniewski, M. B. DonneLLAN y R. E. Lucas (Eds.), Secondary data analysis: An introduction for psychologists (pp. 39-61). Washington, DC: American Psychological Association.

Willems, A., Embregts, P., Stams, G. y Moonen, X. (2010). The relation between intrapersonal and interpersonal staff behaviour towards clients with ID and challenging behaviour: a validation study of the Staff-Client Interactive Behaviour Inventory. Journal of Intellectual Disability Research, 54, 40-51.

Williams, R. y Rose, J. (2007). The development of a questionnaire to assess the perceptions of care staff towards people with intellectual disabilities who display challenging behaviour. Journal of Intellectual Disability, 11 (2), 197-211.

(C) Ediciones Universidad de Salamanca / CC BY-NC-ND

Siglo Cero, vol. 47 (2), n. ${ }^{\circ}$ 258, 2016, abril-junio, pp. 7-41 
EVALUACIÓN DE ACTITUDES DE LOS PROFESIONALES HACIA LAS PERSONAS CON DISCAPACIDAD

V. ARIAS GONZÁLEZ, B. ARIAS MARTÍNEZ, M. Á. VERDUGO ALONSO, M. RUBIA AVI Y C. JENARO RÍO

World Health Organization (2011). World Report on Disability 2011. Geneva: World Health Organization Press.

Yuker, H. E., Block, J. R. y Younng, J. H. (1960). The measurement of attitudes toward disabled persons. Albertson, NY: Human Resources Center.

Zinbarg, R. E., Revelle, W., Yovel, I. y Li, W. (2005). Cronbach's $\alpha$, Revelle's $\beta$, and McDonald's $\omega_{\mathrm{H}}$ : Their relations with each other and two alternative conceptualizations of reliability. Psychometrika, 70, 123-133.

(C) Ediciones Universidad de Salamanca / CC BY-NC-ND

Siglo Cero, vol. 47 (2), n. ${ }^{\circ}$ 258, 2016, abril-junio, pp. 7-41 


\section{APÉNDICE A. Escala de Actitudes}

\section{Escala de actitudes hacia las personas con discapacidad}

(C) 1994, 2000, 2016 M. Á. Verdugo, B. Arias y C. Jenaro (Universidad de Salamanca)

Este cuestionario pretende recoger información acerca del signo de las actitudes de los profesionales de diversos ámbitos hacia la discapacidad, fundamentales para el diseño de actuaciones futuras. Le agradeceremos que dedique un poco de su tiempo para contestarlo.

Para anotar sus respuestas a cada pregunta, basta con que señale el código asociado a su situación personal o respuesta elegida (Ejemplo: si Vd. es varón deberá rodear con un círculo el código 2 en la pregunta 1$)$.

1. Sexo

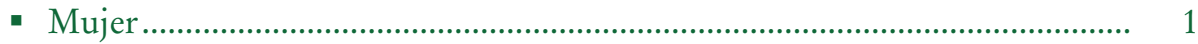

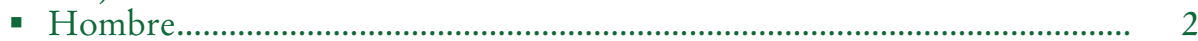

2. Edad

- Hasta 20 ........................................................................................................ 1

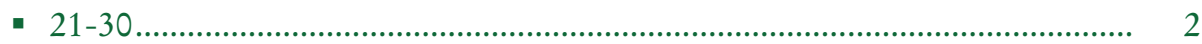

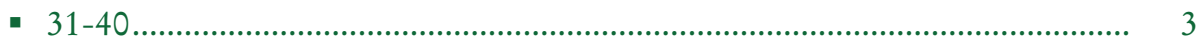

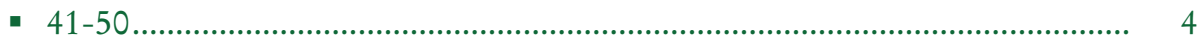

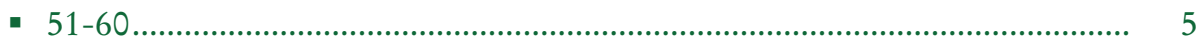

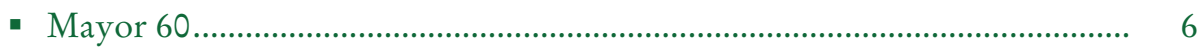

3. Nivel de estudios

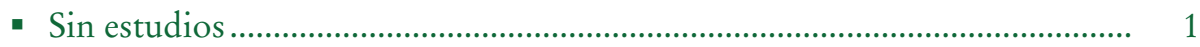

- Primarios........................................................................................... 2

- Bachillerato .............................................................................................. 3

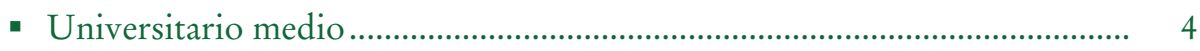

- Universitario superior ................................................................................. 5

4. Profesión o trabajo habitual

5. ¿Mantiene algún tipo de contacto con personas con discapacidad?

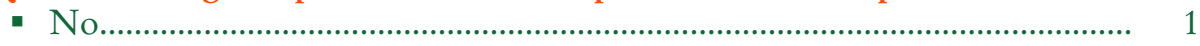

- Sí .................................................................................................................... 2

En caso afirmativo, responda por favor a P.6 (a, b y c)

6.a. Razón del contacto (puede señalar más de una)

- Familiar

- Laboral. 
- Asistencial

- Ocio o amistad

- Otras razones

6.b. Frecuencia del contacto

- Todos los días

- Varias veces por semana ............................................................................... 2

- Varias veces al mes ............................................................................................. 3

- Menos de una vez al mes .............................................................................. 4

6.c. Tipo de discapacidad (puede señalar más de una)

- Física

- Auditiva

- Visual.

- Intelectual

- Enfermedad mental

- Múltiple

7. En general, ¿̨cómo se siente en presencia de una persona con discapacidad?

- Muy cómodo.....

- Bastante cómodo

- Indiferente

- Bastante incómodo

- Muy incómodo.

8. ¿En qué medida se siente informado sobre las diferentes discapacidades?

\section{8.a. Discapacidad física}

- Bien informado

- Poco informado.

\section{8.b. Discapacidad psíquica}

- Bien informado

- Poco informado.

\section{8.c. Discapacidad sensorial}

- Bien informado.

- Poco informado. 


\section{ESCALA DE ACTITUDES}

A continuación le planteamos una serie de afirmaciones. Le pedimos su grado de acuerdo para cada una de ellas. En dichas frases se utiliza la expresión personas con discapacidad para referirnos de manera general a todas aquellas personas que presentan alguna deficiencia, limitación en sus actividades o restricción en su participación social. Las discapacidades más comunes son: las procedentes de deficiencias físicas, deficiencias auditivas, deficiencias visuales, discapacidad intelectual (anteriormente conocida como retraso mental), la enfermedad mental y la presencia en la misma persona de varias de esas deficiencias. Quedan excluidas aquellas personas cuya discapacidad es producto de su elevada edad.

Su tarea consiste en opinar si está de acuerdo o no con cada una de las frases que se le van a presentar, teniendo en cuenta que:

(a) No existen respuestas buenas o malas, correctas o erróneas; cada opción indica simplemente una diferente forma de pensar.

(b) Procure contestar a todas las frases, incluso a aquellas que no se ajusten a sus circunstancias concretas.

(c) En caso de duda entre varias opciones, señale aquella que se acerque más a su forma de pensar. Circule siempre una sola respuesta por frase.

(d) Lea con atención cada frase, pero no se demore demasiado en señalar su respuesta.

(e) Conteste con sinceridad. El cuestionario es totalmente confidencial y anónimo y sus respuestas sólo se tratarán estadísticamente de forma conjunta con el resto de personas que respondan.

\begin{tabular}{|c|l|c|c|c|c|}
\hline \multicolumn{2}{|l|}{} & $\begin{array}{c}\text { Muy de } \\
\text { acuerdo }\end{array}$ & $\begin{array}{c}\text { Bastante } \\
\text { acuerdo }\end{array}$ & $\begin{array}{c}\text { Bastante } \\
\text { desacuerdo }\end{array}$ & $\begin{array}{c}\text { Muy } \\
\text { desacuerdo }\end{array}$ \\
\hline $\mathbf{1}$ & $\begin{array}{l}\text { Las personas con discapacidad pueden } \\
\text { sentirse tan satisfechas de sí mismas } \\
\text { como cualquier persona. }\end{array}$ & 4 & 3 & 2 & 1 \\
\hline $\mathbf{2}$ & $\begin{array}{l}\text { Las personas con discapacidad } \\
\text { deberían tener las mismas } \\
\text { oportunidades de empleo que } \\
\text { cualquier persona. }\end{array}$ & 4 & 3 & 2 & 1 \\
\hline $\mathbf{3}$ & $\begin{array}{l}\text { Las personas con discapacidad } \\
\text { son menos productivas en su lugar } \\
\text { de trabajo que las personas sin } \\
\text { discapacidad. }\end{array}$ & 1 & 2 & 3 & 4 \\
\hline $\mathbf{5}$ & $\begin{array}{l}\text { Debería hacerse algo para conseguir } \\
\text { una mayor integración de las personas } \\
\text { con discapacidad, por ejemplo, } \\
\text { facilitándoles el acceso a los lugares } \\
\text { públicos. }\end{array}$ & 4 & 3 & 2 & 1 \\
\hline $\mathbf{6}$ & $\begin{array}{l}\text { Las personas con discapacidad severa } \\
\text { deberían ser confinadas en centros } \\
\text { especiales. }\end{array}$ & 1 & 2 & 3 & 4 \\
\hline
\end{tabular}




\begin{tabular}{|c|c|c|c|c|c|}
\hline & & $\begin{array}{l}\text { Muy de } \\
\text { acuerdo }\end{array}$ & $\begin{array}{l}\text { Bastante } \\
\text { acuerdo }\end{array}$ & $\begin{array}{c}\text { Bastante } \\
\text { desacuerdo }\end{array}$ & $\begin{array}{c}\text { Muy } \\
\text { desacuerdo }\end{array}$ \\
\hline 7 & $\begin{array}{l}\text { En situaciones sociales, preferiría } \\
\text { no encontrarme con personas con } \\
\text { discapacidad. }\end{array}$ & 1 & 2 & 3 & 4 \\
\hline 8 & \begin{tabular}{|l|} 
Las personas con discapacidad son tan \\
valiosas como cualquiera.
\end{tabular} & 4 & 3 & 2 & 1 \\
\hline 9 & $\begin{array}{l}\text { En general me siento a disgusto } \\
\text { en compañía de una persona con } \\
\text { discapacidad. }\end{array}$ & 1 & 2 & 3 & 4 \\
\hline 10 & \begin{tabular}{|l|} 
Si me citara con una persona con \\
discapacidad, me preocuparía lo que la \\
gente pudiera pensar.
\end{tabular} & 1 & 2 & 3 & 4 \\
\hline 11 & $\begin{array}{l}\text { Las personas con discapacidad son } \\
\text { capaces de adaptarse a una vida } \\
\text { independiente. } \\
\end{array}$ & 4 & 3 & 2 & 1 \\
\hline 13 & $\begin{array}{l}\text { Si tuviera un familiar cercano con } \\
\text { discapacidad, evitaría comentarlo con } \\
\text { otras personas. }\end{array}$ & 1 & 2 & 3 & 4 \\
\hline 14 & $\begin{array}{l}\text { Las personas con discapacidad } \\
\text { deberían poder divertirse con el resto } \\
\text { de las personas. }\end{array}$ & 4 & 3 & 2 & 1 \\
\hline 15 & $\begin{array}{l}\text { Las personas con discapacidad pueden } \\
\text { disfrutar del deporte tanto como } \\
\text { cualquier otra persona. }\end{array}$ & 4 & 3 & 2 & 1 \\
\hline 16 & $\begin{array}{l}\text { Creo que me resultaría fácil } \\
\text { relacionarme con una persona con } \\
\text { discapacidad. }\end{array}$ & 4 & 3 & 2 & 1 \\
\hline 18 & $\begin{array}{l}\text { Si tuviera que hablar con una persona } \\
\text { con discapacidad, me preocuparía no } \\
\text { saber cómo comportarme. }\end{array}$ & 1 & 2 & 3 & 4 \\
\hline 19 & $\begin{array}{l}\text { Podría citarme con una persona con } \\
\text { discapacidad con la misma facilidad } \\
\text { que con cualquier otra persona. }\end{array}$ & 4 & 3 & 2 & 1 \\
\hline 20 & $\begin{array}{l}\text { Las personas con discapacidad } \\
\text { pueden alcanzar un elevado nivel de } \\
\text { autodeterminación e independencia. }\end{array}$ & 4 & 3 & 2 & 1 \\
\hline 21 & $\begin{array}{l}\text { Me gustaría que mi hijo se relacionara } \\
\text { con niños con discapacidad. }\end{array}$ & 4 & 3 & 2 & 1 \\
\hline 22 & $\begin{array}{l}\text { Los trabajadores con discapacidad } \\
\text { deberían recibir el mismo salario que } \\
\text { los que no tienen discapacidad. }\end{array}$ & 4 & 3 & 2 & 1 \\
\hline 23 & $\begin{array}{l}\text { Las personas con discapacidad tienen } \\
\text { los mismos derechos que el resto de } \\
\text { las personas. }\end{array}$ & 4 & 3 & 2 & 1 \\
\hline 24 & $\begin{array}{l}\text { Evitaría acompañar a una persona con } \\
\text { discapacidad a comer a un restaurante } \\
\text { en el que me conocieran. }\end{array}$ & 1 & 2 & 3 & 4 \\
\hline
\end{tabular}




\begin{tabular}{|c|c|c|c|c|c|}
\hline & & $\begin{array}{l}\text { Muy de } \\
\text { acuerdo }\end{array}$ & $\begin{array}{l}\text { Bastante } \\
\text { acuerdo }\end{array}$ & $\begin{array}{c}\text { Bastante } \\
\text { desacuerdo }\end{array}$ & $\begin{array}{c}\text { Muy } \\
\text { desacuerdo }\end{array}$ \\
\hline 25 & $\begin{array}{l}\text { El comportamiento de las personas } \\
\text { con discapacidad en general es } \\
\text { irritante. }\end{array}$ & 1 & 2 & 3 & 4 \\
\hline 26 & $\begin{array}{l}\text { Los programas de rehabilitación para } \\
\text { las personas con discapacidad son } \\
\text { excesivamente costosos para lo poco } \\
\text { que se consigue. }\end{array}$ & 1 & 2 & 3 & 4 \\
\hline 27 & $\begin{array}{l}\text { Los niños con discapacidad deberían } \\
\text { acudir a los mismos colegios que los } \\
\text { demás niños. }\end{array}$ & 4 & 3 & 2 & 1 \\
\hline 28 & $\begin{array}{l}\text { No me importaría tener como amiga a } \\
\text { una persona con discapacidad. } \\
\end{array}$ & 4 & 3 & 2 & 1 \\
\hline 29 & $\begin{array}{l}\text { Las personas con discapacidad pueden } \\
\text { tener una personalidad tan equilibrada } \\
\text { como las personas sin discapacidad. }\end{array}$ & 4 & 3 & 2 & 1 \\
\hline 30 & $\begin{array}{l}\text { Las personas con discapacidad son } \\
\text { tan amistosas como cualquier otra } \\
\text { persona. }\end{array}$ & 4 & 3 & 2 & 1 \\
\hline 31 & $\begin{array}{l}\text { Encontrarme con una persona con } \\
\text { discapacidad me produce tensión y } \\
\text { malestar. }\end{array}$ & 1 & 2 & 3 & 4 \\
\hline 32 & $\begin{array}{l}\text { Si tuviera que trabajar con una } \\
\text { persona con discapacidad, me limitaría } \\
\text { a mantener con ella una relación } \\
\text { superficial. }\end{array}$ & 1 & 2 & 3 & 4 \\
\hline 33 & $\begin{array}{l}\text { Debería gastarse más dinero en } \\
\text { suprimir las barreras físicas que siguen } \\
\text { dificultando la vida a las personas con } \\
\text { discapacidad. }\end{array}$ & 4 & 3 & 2 & 1 \\
\hline 34 & $\begin{array}{l}\text { El dinero que se gasta en atender a } \\
\text { las personas con discapacidad debería } \\
\text { destinarse a problemas sociales más } \\
\text { urgentes. }\end{array}$ & 1 & 2 & 3 & 4 \\
\hline \multicolumn{6}{|c|}{ Gracias por su colaboración } \\
\hline
\end{tabular}


EVALUACIÓN DE ACTITUDES DE LOS PROFESIONALES HACIA LAS PERSONAS CON DISCAPACIDAD

V. ARIAS GONZÁLEZ, B. ARIAS MARTÍNEZ, M. Á. VERDUGO ALONSO, M. RUBIA AVI Y C. JENARO RÍO

\section{APÉNDICE B. Baremos}

Cálculo de las puntuaciones derivadas por factor (Ejemplo de un respondiente con puntuaciones directas de 38 en Factor 1, 45 en Factor 2 y 18 en Factor 3).

\begin{tabular}{|c|c|c|c|}
\hline & PD & $\mathrm{T}$ & Centil \\
\hline $\begin{array}{l}\text { Factor 1: } \\
i 07+i 09+i 10+i 13+i 16+i 18+i 19+i 21+i 24+i 25+i 28+i 31+i 32=\end{array}$ & 38 & 34 & 6 \\
\hline $\begin{array}{l}\text { Factor 2: } \\
\mathrm{i} 01+\mathrm{i} 02+\mathrm{i} 03+\mathrm{i} 08+\mathrm{i} 11+\mathrm{i} 14+\mathrm{i} 15+\mathrm{i} 20+\mathrm{i} 22+\mathrm{i} 23+\mathrm{i} 27+\mathrm{i} 29+\mathrm{i} 30=\end{array}$ & 45 & 48 & 42 \\
\hline $\begin{array}{l}\text { Factor 3: } \\
i 05+i 06+i 26+i 33+i 34=\end{array}$ & 18 & 51 & 53 \\
\hline Total $=$ Factor $1+$ Factor $2+$ Factor 3 & 101 & 41 & 19 \\
\hline
\end{tabular}

\begin{tabular}{|c|c|c|c|c|c|c|c|}
\hline \multicolumn{8}{|c|}{ FACTOR 1: Relaciones sociales e interpersonales, RS } \\
\hline $\mathrm{PD}$ & $\mathrm{z}$ perc. & prop. exacta & $\mathrm{z}$ exacta & stanine & sten & $\mathrm{T}$ & Percentil \\
\hline$<28$ & -4.000 & .000 & -4.000 & 1 & 1 & 10 & 0 \\
\hline 28 & -4.000 & .001 & -3.266 & 1 & 1 & 17 & 0 \\
\hline 29 & -4.000 & .002 & -2.940 & 1 & 1 & 21 & 0 \\
\hline 30 & -4.000 & .002 & -2.849 & 1 & 1 & 22 & 0 \\
\hline 31 & -4.000 & .002 & -2.849 & 1 & 1 & 22 & 0 \\
\hline 32 & -4.000 & .002 & -2.849 & 1 & 1 & 22 & 0 \\
\hline 33 & -4.000 & .002 & -2.849 & 1 & 1 & 22 & 0 \\
\hline 34 & -4.000 & .004 & -2.621 & 1 & 1 & 24 & 0 \\
\hline 35 & -2.328 & .010 & -2.310 & 1 & 1 & 27 & 1 \\
\hline 36 & -2.054 & .020 & -2.058 & 1 & 1 & 29 & 2 \\
\hline 37 & -1.881 & .035 & -1.816 & 1 & 2 & 32 & 3 \\
\hline 38 & -1.554 & .057 & -1.577 & 2 & 2 & 34 & 6 \\
\hline 39 & -1.340 & .087 & -1.358 & 2 & 3 & 36 & 9 \\
\hline 40 & -1.173 & .121 & -1.170 & 3 & 3 & 38 & 12 \\
\hline 41 & -1.034 & .153 & -1.023 & 3 & 3 & 40 & 15 \\
\hline 42 & -0.875 & .189 & -0.879 & 3 & 4 & 41 & 19 \\
\hline 43 & -0.736 & .233 & -0.726 & 4 & 4 & 43 & 23 \\
\hline 44 & -0.551 & .287 & -0.559 & 4 & 4 & 44 & 29 \\
\hline 45 & -0.383 & .351 & -0.381 & 4 & 5 & 46 & 35 \\
\hline 46 & -0.226 & .414 & -0.215 & 5 & 5 & 48 & 41 \\
\hline 47 & -0.025 & .486 & -0.034 & 5 & 5 & 50 & 49 \\
\hline 48 & 0.175 & .568 & 0.171 & 5 & 6 & 52 & 57 \\
\hline 49 & 0.410 & .659 & 0.406 & 6 & 6 & 54 & 66 \\
\hline 50 & 0.672 & .749 & 0.667 & 6 & 7 & 57 & 75 \\
\hline 51 & 0.992 & .835 & 0.973 & 7 & 7 & 60 & 84 \\
\hline 52 & 1.554 & .940 & 1.558 & 8 & 9 & 66 & 94 \\
\hline
\end{tabular}

(C) Ediciones Universidad de Salamanca / CC BY-NC-ND

Siglo Cero, vol. 47 (2), n. ${ }^{\circ} 258,2016$, abril-junio, pp. 7-41 
EVALUACIÓN DE ACTITUDES DE LOS PROFESIONALES HACIA LAS PERSONAS CON DISCAPACIDAD

V. ARIAS GONZÁLEZ, B. ARIAS MARTÍNEZ, M. Á. VERDUGO ALONSO, M. RUBIA AVI Y C. JENARO RÍO

\begin{tabular}{|c|c|c|c|c|c|c|c|}
\hline \multicolumn{8}{|c|}{ FACTOR 2: Vida normalizada, VN } \\
\hline PD & $\mathrm{z}$ perc. & prop. exacta & $\mathrm{z}$ exacta & stanine & sten & $\mathrm{T}$ & Percentil \\
\hline$<26$ & -4.000 & .000 & -4.000 & 1 & 1 & 10 & 0 \\
\hline 26 & -4.000 & .001 & -3.259 & 1 & 1 & 17 & 0 \\
\hline 27 & -4.000 & .001 & -3.056 & 1 & 1 & 19 & 0 \\
\hline 28 & -4.000 & .002 & -2.842 & 1 & 1 & 22 & 0 \\
\hline 29 & -4.000 & .003 & -2.710 & 1 & 1 & 23 & 0 \\
\hline 30 & -4.000 & .004 & -2.658 & 1 & 1 & 23 & 0 \\
\hline 31 & -2.328 & .005 & -2.572 & 1 & 1 & 24 & 1 \\
\hline 32 & -2.328 & .006 & -2.502 & 1 & 1 & 25 & 1 \\
\hline 33 & -2.328 & .008 & -2.390 & 1 & 1 & 26 & 1 \\
\hline 34 & -2.328 & .012 & -2.263 & 1 & 1 & 27 & 1 \\
\hline 35 & -2.054 & .017 & -2.123 & 1 & 1 & 29 & 2 \\
\hline 36 & -2.054 & .023 & -2.004 & 1 & 1 & 30 & 2 \\
\hline 37 & -1.881 & .033 & -1.842 & 1 & 2 & 32 & 3 \\
\hline 38 & -1.644 & .054 & -1.610 & 2 & 2 & 34 & 5 \\
\hline 39 & -1.404 & .084 & -1.381 & 2 & 3 & 36 & 8 \\
\hline 40 & -1.125 & .126 & -1.144 & 3 & 3 & 39 & 13 \\
\hline 41 & -0.952 & .169 & -0.955 & 3 & 4 & 40 & 17 \\
\hline 42 & -0.770 & .223 & -0.760 & 3 & 4 & 42 & 22 \\
\hline 43 & -0.551 & .287 & -0.559 & 4 & 4 & 44 & 29 \\
\hline 44 & -0.383 & .349 & -0.386 & 4 & 5 & 46 & 35 \\
\hline 45 & -0.200 & .421 & -0.198 & 5 & 5 & 48 & 42 \\
\hline 46 & 0.000 & .500 & 0.000 & 5 & 6 & 50 & 50 \\
\hline 47 & 0.200 & .584 & 0.211 & 5 & 6 & 52 & 58 \\
\hline 48 & 0.465 & .676 & 0.454 & 6 & 6 & 55 & 68 \\
\hline 49 & 0.736 & .771 & 0.741 & 6 & 7 & 57 & 77 \\
\hline 50 & 1.034 & .852 & 1.044 & 7 & 8 & 60 & 85 \\
\hline 51 & 1.404 & .916 & 1.381 & 8 & 8 & 64 & 92 \\
\hline 52 & 1.881 & .974 & 1.944 & 9 & 9 & 69 & 97 \\
\hline
\end{tabular}

FACTOR 3: Programas de intervención, PI

\begin{tabular}{|c|c|c|c|c|c|c|c|}
\hline PD & z perc. & prop. exacta & z exacta & stanine & sten & T & Percentil \\
\hline$<11$ & -4.000 & .000 & -4.000 & 1 & 1 & 10 & 0 \\
\hline 11 & -4.000 & .003 & -2.717 & 1 & 1 & 23 & 0 \\
\hline 12 & -2.328 & .012 & -2.271 & 1 & 1 & 27 & 1 \\
\hline 13 & -1.881 & .031 & -1.860 & 1 & 2 & 31 & 3 \\
\hline 14 & -1.475 & .072 & -1.461 & 2 & 3 & 35 & 7 \\
\hline 15 & -1.125 & .134 & -1.105 & 3 & 3 & 39 & 13 \\
\hline 16 & -0.704 & .236 & -0.715 & 4 & 4 & 43 & 24 \\
\hline 17 & -0.303 & .376 & -0.313 & 4 & 5 & 47 & 38 \\
\hline
\end{tabular}

() Ediciones Universidad de Salamanca / CC BY-NC-ND

Siglo Cero, vol. 47 (2), n. ${ }^{\circ} 258,2016$, abril-junio, pp. 7-41 
EVALUACIÓN DE ACTITUDES DE LOS PROFESIONALES HACIA LAS PERSONAS CON DISCAPACIDAD

V. ARIAS GONZÁLEZ, B. ARIAS MARTÍNEZ, M. Á. VERDUGO ALONSO, M. RUBIA AVI Y C. JENARO RÍO

\begin{tabular}{|c|c|c|c|c|c|c|c|}
\hline \multicolumn{7}{|c|}{ FACTOR 3: Programas de intervención, PI } \\
\hline PD & z perc. & prop. exacta & z exacta & stanine & sten & T & Percentil \\
\hline 18 & 0.074 & .534 & 0.084 & 5 & 6 & 51 & 53 \\
\hline 19 & 0.522 & .704 & 0.534 & 6 & 7 & 55 & 70 \\
\hline 20 & 1.280 & .896 & 1.258 & 8 & 8 & 63 & 90 \\
\hline
\end{tabular}

\begin{tabular}{|c|c|c|c|c|c|c|c|}
\hline \multicolumn{8}{|c|}{ Escala Total } \\
\hline PD & $\mathrm{z}$ perc. & prop. exacta & z exacta & stanine & sten & $\mathrm{T}$ & Percentil \\
\hline$<78$ & -4.000 & .000 & -4.000 & 1 & 1 & 10 & 0 \\
\hline 78 & -4.000 & .001 & -3.232 & 1 & 1 & 18 & 0 \\
\hline 79 & -4.000 & .002 & -2.903 & 1 & 1 & 21 & 0 \\
\hline 80 & -4.000 & .002 & -2.812 & 1 & 1 & 22 & 0 \\
\hline 81 & -4.000 & .004 & -2.679 & 1 & 1 & 23 & 0 \\
\hline 82 & -2.328 & .006 & -2.540 & 1 & 1 & 25 & 1 \\
\hline 83 & -2.328 & .007 & -2.468 & 1 & 1 & 25 & 1 \\
\hline 84 & -2.328 & .008 & -2.408 & 1 & 1 & 26 & 1 \\
\hline 85 & -2.328 & .011 & -2.308 & 1 & 1 & 27 & 1 \\
\hline 86 & -2.328 & .013 & -2.227 & 1 & 1 & 28 & 1 \\
\hline 87 & -2.328 & .014 & -2.191 & 1 & 1 & 28 & 1 \\
\hline 88 & -2.054 & .017 & -2.127 & 1 & 1 & 29 & 2 \\
\hline 89 & -2.054 & .021 & -2.033 & 1 & 1 & 30 & 2 \\
\hline 90 & -1.881 & .027 & -1.933 & 1 & 2 & 31 & 3 \\
\hline 91 & -1.751 & .037 & -1.792 & 1 & 2 & 32 & 4 \\
\hline 92 & -1.644 & .051 & -1.637 & 2 & 2 & 34 & 5 \\
\hline 93 & -1.554 & .061 & -1.543 & 2 & 2 & 35 & 6 \\
\hline 94 & -1.475 & .069 & -1.484 & 2 & 3 & 35 & 7 \\
\hline 95 & -1.404 & .079 & -1.409 & 2 & 3 & 36 & 8 \\
\hline 96 & -1.340 & .089 & -1.344 & 2 & 3 & 37 & 9 \\
\hline 97 & -1.280 & .100 & -1.278 & 2 & 3 & 37 & 10 \\
\hline 98 & -1.173 & .122 & -1.163 & 3 & 3 & 38 & 12 \\
\hline 99 & -1.078 & .144 & -1.059 & 3 & 3 & 39 & 14 \\
\hline 100 & -0.992 & .164 & -0.975 & 3 & 4 & 40 & 16 \\
\hline 101 & -0.875 & .193 & -0.863 & 3 & 4 & 41 & 19 \\
\hline 102 & -0.770 & .222 & -0.761 & 3 & 4 & 42 & 22 \\
\hline 103 & -0.672 & .247 & -0.683 & 4 & 4 & 43 & 25 \\
\hline 104 & -0.610 & .271 & -0.608 & 4 & 4 & 44 & 27 \\
\hline 105 & -0.522 & .298 & -0.527 & 4 & 4 & 45 & 30 \\
\hline 106 & -0.437 & .330 & -0.438 & 4 & 5 & 46 & 33 \\
\hline 107 & -0.356 & .365 & -0.343 & 4 & 5 & 47 & 36 \\
\hline 108 & -0.251 & .401 & -0.247 & 5 & 5 & 48 & 40 \\
\hline
\end{tabular}

() Ediciones Universidad de Salamanca / CC BY-NC-ND

Siglo Cero, vol. 47 (2), n. ${ }^{\circ} 258,2016$, abril-junio, pp. 7-41

$$
-40-
$$


EVALUACIÓN DE ACTITUDES DE LOS PROFESIONALES HACIA LAS PERSONAS CON DISCAPACIDAD

V. ARIAS GONZÁlEZ, B. ARIAS MARTÍNEZ, M. Á. VERDUGO ALONSO, M. RUBIA AVI Y C. JENARO RÍO

\begin{tabular}{|c|c|c|c|c|c|c|c|}
\hline \multicolumn{7}{|c|}{ Escala Total } \\
\hline PD & z perc. & prop. exacta & z exacta & stanine & sten & T & Percentil \\
\hline 109 & -0.150 & .443 & -0.142 & 5 & 5 & 49 & 44 \\
\hline 110 & -0.025 & .486 & -0.034 & 5 & 5 & 50 & 49 \\
\hline 111 & 0.050 & .523 & 0.057 & 5 & 6 & 51 & 52 \\
\hline 112 & 0.150 & .557 & 0.142 & 5 & 6 & 51 & 56 \\
\hline 113 & 0.226 & .595 & 0.238 & 5 & 6 & 52 & 59 \\
\hline 114 & 0.356 & .637 & 0.348 & 6 & 6 & 53 & 64 \\
\hline 115 & 0.465 & .682 & 0.469 & 6 & 6 & 55 & 68 \\
\hline 116 & 0.610 & .726 & 0.597 & 6 & 7 & 56 & 73 \\
\hline 117 & 0.704 & .765 & 0.718 & 6 & 7 & 57 & 76 \\
\hline 118 & 0.839 & .804 & 0.854 & 7 & 7 & 59 & 80 \\
\hline 119 & 0.992 & .842 & 1.001 & 7 & 8 & 60 & 84 \\
\hline 120 & 1.173 & .879 & 1.169 & 7 & 8 & 62 & 88 \\
\hline 121 & 1.404 & .921 & 1.409 & 8 & 8 & 64 & 92 \\
\hline 122 & 1.751 & .955 & 1.699 & 8 & 9 & 67 & 96 \\
\hline 123 & 2.054 & .978 & 2.021 & 9 & 10 & 70 & 98 \\
\hline 124 & 2.328 & .994 & 2.502 & 9 & 10 & 75 & 99 \\
\hline
\end{tabular}

